\title{
Detecting shocked intergalactic gas with X-ray and radio observations
}

\author{
F. Vazza ${ }^{1,2,3}$, S. Ettori ${ }^{4,5}$, M. Roncarelli ${ }^{1,4}$, M. Angelinelli ${ }^{1}$, M. Brüggen ${ }^{2}$, and C. Gheller $^{6}$ \\ 1 Dipartimento di Fisica e Astronomia, Universitá di Bologna, Via Gobetti 93/2, 40122 Bologna, Italy \\ e-mail: franco.vazza@hs .uni-hamburg.de \\ 2 Hamburger Sternwarte, Gojenbergsweg 112, 21029 Hamburg, Germany \\ 3 Istituto di Radioastronomia, INAF, Via Gobetti 101, 40122 Bologna, Italy \\ 4 INAF, Osservatorio di Astrofisica e Scienza dello Spazio, Via Pietro Gobetti 93/3, 40129 Bologna, Italy \\ 5 INFN, Sezione di Bologna, Viale Berti Pichat 6/2, 40127 Bologna, Italy \\ 6 Swiss Plasma Center, EPFL, SB SPC Station 13, 1015 Lausanne, Switzerland
}

Received 9 March 2019 / Accepted 8 May 2019

\begin{abstract}
Detecting the thermal and non-thermal emission from the shocked cosmic gas surrounding large-scale structures represents a challenge for observations, as well as a unique window into the physics of the warm-hot intergalactic medium. In this work, we present synthetic radio and X-ray surveys of large cosmological simulations in order to assess the chances of jointly detecting the cosmic web in both frequency ranges. We then propose best observing strategies tailored for existing (LOFAR, MWA, and XMM) or future instruments (SKA-LOW and SKA-MID, Athena, and eROSITA). We find that the most promising targets are the extreme peripheries of galaxy clusters in an early merging stage, where the merger causes the fast compression of warm-hot gas onto the virial region. By taking advantage of a detection in the radio band, future deep X-ray observations will probe this gas in emission, and help us to study plasma conditions in the dynamic warm-hot intergalactic medium with unprecedented detail.
\end{abstract}

Key words. shock waves - X-rays: galaxies: clusters - large-scale structure of Universe - galaxies: clusters: intracluster medium methods: numerical

\section{Introduction}

Numerical simulations (e.g. Cen \& Ostriker 1999; Davé et al. 2001; Gheller et al. 2016; Martizzi et al. 2019) have shown that the most important mass component of the baryons in the cosmic web is the elusive warm-hot intergalatic medium (WHIM), a rarefied gas with densities $n \sim 10^{-5}-10^{-4}$ part $\mathrm{cm}^{-3}$ and temperatures $T \sim 10^{5}-10^{7} \mathrm{~K}$. The WHIM most likely fills the volume within cosmic filaments as well as in the outskirts of galaxy clusters, and attains its temperature via strong $(\mathcal{M} \gg 10)$ accretion shocks.

Detections of absorption lines through the WHIM of intracluster filaments have been claimed (e.g. Werner et al. 2008; Nicastro et al. 2010). More recently, Nicastro et al. (2018) reported the possible detection of two OVII absorbers in the X-ray spectrum of a quasar at $z \geq 0.4$, possibly tracing the WHIM.

In a few nearby galaxy clusters and limited to a few narrow sectors, the thermodynamical properties of the intracluster medium have been mapped with X-ray observations out to $R_{100}$ (e.g. Simionescu et al. 2011; Urban et al. 2011). Others mapped the gas properties in concentric rings out to $R_{200}$ by combining X-ray and Sunyaev-Zeldovich (SZ) data (Ghirardini et al. 2019; Ettori et al. 2019; Eckert et al. 2019). The ends of five massive filaments connected to the massive cluster A2744 have been observed with XMM-Newton (Eckert et al. 2015), possibly representing the first images of cosmic filaments in the X-ray band. This made it possible to estimate that $5-10 \%$ of the mass fraction of missing baryons may be bound in such objects.

Moreover, the study of the Sunyaev-Zeldovich effect from the outer region of clusters, either in single pointings of interacting clusters (Planck Collaboration VIII 2013; Bonjean et al. 2018) or in stacked observations of larger samples (Tanimura et al. 2019; de Graaff et al. 2019) has detected the hot $\left(\sim 10^{7} \mathrm{~K}\right)$ and very over-dense $\left(\sim 10-10^{2} \rho /\langle\rho\rangle\right)$ gas component, potentially contributing to $\sim 10-50 \%$ of missing cosmic baryons.

Cosmological hydrodynamical simulations have shown that the WHIM follows the underlying galaxy distribution (see e.g. Nevalainen et al. 2015) and that its diffuse emission is responsible for a significant fraction of the unresolved X-ray background in very deep Chandra observations (see Roncarelli et al. 2006a; Hickox \& Markevitch 2007), with a predicted surface brightness on the order of $1-5 \times 10^{13} \mathrm{erg} /\left(\mathrm{cm}^{2} \mathrm{~s} \mathrm{deg}^{2}\right)$ in the $\sim 0.5-1 \mathrm{keV}$ energy band, with uncertainties related to its metal composition (Ursino et al. 2010; Cen \& Chisari 2011; Roncarelli et al. 2012). However, the systematic detection and characterization of single WHIM systems remain a challenge due to its low emissivity. Hence, efforts have focused on stacking and statistical studies on the (auto)correlation function of its X-ray signal (see e.g. Piro et al. 2009; Takei et al. 2011; Ursino et al. 2011; Cappelluti et al. 2012; Kolodzig et al. 2018).

The planned X-ray mission Athena X-ray observatory ${ }^{1}$, expected to be launched by $\sim 2030$, holds great promise to detect the WHIM in absorption. Among its ambitious goals, Athena aims to trace the missing baryons in the intergalactic medium via detecting their absorption lines, through the emission of bright sources up to $z \sim 2$. It is expected that in total $\sim 80$ sources can be studied at the highest possible resolution for spectroscopic studies with the instrument X-IFO $(\Delta E=2.5 \mathrm{eV})$ (Barcons et al. 2012).

1 http://www . the-athena-x-ray-observatory.eu 
The limitation of this technique is that, of course, most of these sources are unpredictable and variable, and only a dozen bright enough sources per year may be detected to study filaments. Concepts for future high-resolution X-ray imagers, capable of further reducing the limiting effect of the X-ray background from unresolved point-like sources, have recently been presented in the context of the US Decadal Survey (e.g. Lynx The Lynx Team 2018, AXIS Mushotzky et al. 2019). In this work, we wish to explore a complementary approach, in which Athena detects the WHIM in emission.

Planelles et al. (2018) have recently presented detailed radiative transfer calculations of the X-ray emission from a cluster at $z \approx 0.3$, reporting that the emission from the WHIM only accounts for $\sim 5 \%$ in the $[0.5-2] \mathrm{keV}$ band and $\sim 1 \%$ in the [2-10] keV band. In general, the WHIM is found to have a more filamentary structure than the $\geq 10^{7} \mathrm{~K}$ gas phase, extending several Megaparsecs (Mpc) out from the virial regions of galaxy clusters. Using a larger volume with the Illustris-The Next Generation (TNG) suite, Martizzi et al. (2019) recently studied the WHIM properties from $z=4$ to $z=0$, confirming that most of the filaments are more baryon-rich than the cosmic average, but that they have a significantly lower metallicity than the Intra Cluster Medium (ICM), which makes their observability via $\mathrm{X}$-ray observations challenging.

In addition to the $\mathrm{X}$-ray window, also the radio window may be able to image the cosmic web, thanks to the current (e.g. the Low Frequency Array (LOFAR), the Murchison Widefield Array (MWA), the Australian Square Kilometre Array Pathfinder (ASKAP), MeerKAT) and future (e.g. the Square Kilometer Array, SKA) generations of large radio telescopes. Cosmological simulations have shown that filaments of the cosmic web are surrounded by strong and quasi-stationary accretion shocks (e.g. Ryu et al. 2003; Pfrommer et al. 2006; Vazza et al. 2011), at which a tiny fraction of relativistic electrons may be accelerated. This is similar to what occurs in radio relics or cluster radio shocks at the periphery of clusters (e.g. Hoeft \& Brüggen 2007; Wittor et al. 2017; van Weeren et al. 2019).

A few radio observations have already claimed the detection of diffuse synchrotron emission from the shocked gas at the interface between galaxy clusters and filaments attached to them (Bagchi et al. 2002; Giovannini et al. 2010; Farnsworth et al. 2013; Vacca et al. 2018). Moreover, the observation of Faraday Rotation by filaments in the Coma cluster has been claimed (Bonafede et al. 2013). Recently, the signature in Faraday space of filaments overlapping with the emission of a $z=0.34$ radio galaxy has been claimed by O'Sullivan et al. (2019). The detection of faint diffuse radio emission at the interface of pre-merger galaxy clusters has been reported using LOFAR-High Band Array (HBA) by Botteon et al. (2018) and Govoni et al. (2019).

Simulations have shown that the low surface brightness $\left(\leq \mu \mathrm{Jy} \operatorname{arcsec}^{-2}\right.$ at $\left.\sim 100 \mathrm{MHz}\right)$, highly polarized $(\sim 70 \%)$, and large angular scale $\left(\geq 1^{\circ}\right)$ emission that is expected to be produced by the shocked cosmic web (e.g. Keshet et al. 2004; Brown 2011; Vazza et al. 2015b) makes the low-frequency radio spectrum $(v \leq 300 \mathrm{MHz})$ the most suitable for a detection, owing to the typically superior sampling of short baselines in lowfrequency radio telescopes. In particular, the radio continuum surveys of SKA-LOW should detect parts of the magnetic cosmic web, with statistics depending on the (unknown) details of particle acceleration and magnetic field distribution in such rarefied plasma (e.g. Vazza et al. 2015a,b, 2017a). Additional to this, also polarization surveys with the SKA-MID may be able to detect the Faraday Rotation signal from the terminal part of filaments connected to massive galaxy clusters, provided that a large statistics of polarized sources is available (Locatelli et al. 2018).

The exciting possibility of detecting both thermal and nonthermal emission from the cosmic web with joint X-ray and radio surveys is the subject of this work. In a pilot study for the "SKA-Athena Synergy White Paper" (Cassano et al. 2018, Sect. 5.2.1) we first investigated the potential for a synergy between SKA and Athena in the study of the rarefied cosmic web. Our first results suggest that cluster outskirts are promising targets, with a small but non-negligible fraction of the cosmic web that might be detectable by both instruments (working at their nominal maximum capabilities). This possibility will make it possible to study the WHIM with Athena not only via absorption lines towards high- $z$ powerful sources, but also on a few, carefully selected objects. Based on these results, we use one of the largest cosmological magneto-hydrodynamical simulations ever produced to assess to what extent future X-ray and radio observations can constrain the physical properties of the WHIM.

The structure of the paper is as follows: in Sect. 2 we introduce our simulations, and in particular in Sect. 2.2 we introduce our methods to produce sky models of our simulated universes. In Sect. 3 we present our results for the intrinsic emission properties of the cosmic web in X-ray and radio bands, while in Sect. 3.3.3 we specifically investigate mock Athena and SKA observations of our fields, and in Sect. 3.3.4 we present preliminary simulations of future spectroscopic analyses with the X-ray Integral Field Unit (X-IFU). Physical and numerical limitations of our results are given in Sect. 4 before we conclude in Sect. 5 .

\section{Methods}

\subsection{Cosmological simulations}

As in previous work (Vazza et al. 2017a, 2018a), we simulated a comoving $100^{3} \mathrm{Mpc}^{3}$ box with a uniform grid of $2400^{3}$ cells and $2400^{3}$ dark matter particles, using the cosmological Magneto-Hydordinamical (MHD) code ENZO ${ }^{2}$ (Bryan et al. 2014). The fixed (comoving) spatial resolution of this run is $\Delta x=41.6 \mathrm{kpc}$ cell $^{-1}$ while the fixed dark matter mass resolution is $m_{\mathrm{dm}}=8.62 \times 10^{6} M_{\odot}$ per particle. We initialized magnetic fields at $z=45$ as a simple uniform background of $B_{0}=0.1 \mathrm{nG}$ (one order of magnitude below the upper limits on primordial magnetic fields from the analysis of the Cosmic Microwave Background (CMB), Planck Collaboration XIX 2015), and we used the MHD method of Dedner (Dedner et al. 2002), ported to Graphic Processing Units (Wang et al. 2010) to evolve magnetic fields at run-time.

Our run is non-radiative and does not include any treatment for star formation or feedback from active galactic nuclei. To a first approximation, these processes are not very relevant for the radio and X-ray properties of the peripheral regions of galaxy clusters and filaments, which are our main focus (see Sect. 4 for a discussion).

We assumed a $\Lambda \mathrm{CDM}$ cosmological model, with density parameters $\Omega_{\mathrm{BM}}=0.0455, \Omega_{\mathrm{DM}}=0.2265$ (BM and DM indicating the baryonic and the dark matter respectively), $\Omega_{\Lambda}=0.728$, and a Hubble constant $H_{0}=70.2 \mathrm{~km} /(\mathrm{s} \mathrm{Mpc})$. In Sect. 3.3.4 we will also present results for the resimulation of a massive galaxy cluster with the same setup, but using nested initial conditions and adaptive mesh refinement (eight levels) to achieve a higher resolution $\left(\Delta x_{8} \approx 4 \mathrm{kpc}\right.$ cell $\left.^{-1}\right)$, similar to Vazza et al. (2018b). An impression of the three-dimensional distribution of magnetic

WWW.enzo-project.org 


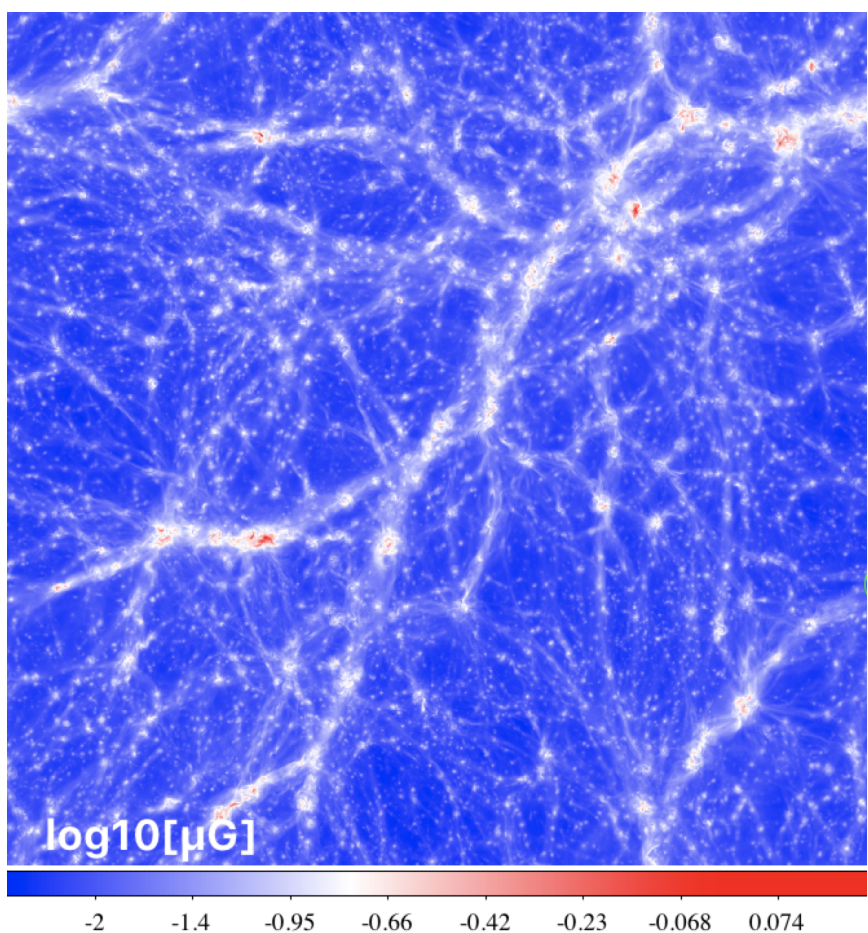

Fig. 1. Projected (mass weighted) magnetic field strength at $z=0.05$ for our simulated $100^{3} \mathrm{Mpc}^{3}$ volume.

fields in our simulation (which is one of the biggest MHD simulations ever performed in cosmology) is given in Fig. 1, and shows the variety of magnetic field strengths and configurations at $z=0.05$.

\subsection{Sky models}

\subsubsection{X-ray emission}

For the X-ray emission, we assumed for simplicity a single temperature and a single (constant) composition for every cell in the simulation, and we computed the emissivity from the B-Astrophysical Plasma Emission Code (B-APEC) ${ }^{3}$, assuming ionization equilibrium and including continuum and line emission. We consider a constant metallicity across the volume, $Z / Z_{\odot}=0.3$ (see the discussion in Sect. 4 on the rather small impact of metallicity in most of our estimates). For each energy band, we computed the cell's X-ray emissivity, $S_{\mathrm{X}}=$ $n_{\mathrm{H}} n_{\mathrm{e}} \Lambda(T, Z) \mathrm{d} V$, where $n_{\mathrm{H}}$ and $n_{\mathrm{e}}$ are the number density of hydrogen and electrons (assuming a primordial composition) respectively, and $\mathrm{d} V$ is the constant volume of our cells.

We do not include the additional contribution from the inverse Compton emission from the same relativistic electrons accelerated by shocks and responsible for the radio emission (see next section), whose amplitude depends on the assumed electron energy distribution at low energies (e.g. Bartels et al. 2015). However, our estimates show that the inverse Compton in the $[0.8-1.2] \mathrm{keV}$ band is negligible $(\leq 1 \%)$ compared to the thermal emission of the cluster. It may start to dominate only at very large radii, $\geq 2-3 R_{100}$, at which no detection seems to be feasible with realistic exposure times.

The left panel of Fig. 2 shows the integrated X-ray emission from the simulated box located at $z=0.05$, in the $[0.8-1.2] \mathrm{keV}$

\footnotetext{
https://heasarc.gsfc.nasa.gov/xanadu/xspec/manual/ Models.html
}

energy band. At the angular distance corresponding to this redshift $\left(D_{\mathrm{A}} \approx 201.6 \mathrm{Mpc}\right)$ this volume covers $28.4^{\circ} \times 28.4^{\circ}$. For reference, Athena's Wide Field Imager field of view is $\sim 40^{\prime} \times 40^{\prime}$, while X-IFU's field of view is $\sim 5^{\prime} \times 5^{\prime}$.

\subsubsection{Radio}

We predict the synchrotron radio emission assuming that diffusive shock acceleration (DSA, e.g. Kang et al. 2012, and references therein) is able to accelerate a very small fraction of thermal electrons swept by shocks up to relativistic energies $\left(\gamma \geq 10^{3}-10^{4}\right)$, and that the intergalactic medium has a nonnegligible magnetic field, as suggested by our MHD simulation (e.g. Fig. 1). As in previous work (Vazza et al. 2015b), we identify shocks in the simulation in post-processing with a velocity-based approach, and we compute the radio emission from electrons accelerated in the shock downstream following Hoeft \& Brüggen (2007). The typical efficiency $\left(\xi_{\mathrm{e}}\right)$ considered in the conversion efficiency from shock kinetic energy into the energy of relativistic electrons is small and it scales with the Mach number and the upstream gas temperature as in Hoeft \& Brüggen (2007): for example it is $\xi_{\mathrm{e}} \approx 10^{-6}$ for $\mathcal{M}=3$ shocks in a $T=10^{7} \mathrm{~K}$, and $\xi_{\mathrm{e}} \approx 6 \times 10^{-4}$ for $\mathcal{M} \geq 50$ shocks with a $T=10^{5} \mathrm{~K}$. The additional (possible) role of shock obliquity (e.g. Wittor et al. 2017) and of fossil reaccelerated electrons (e.g. Pinzke et al. 2013) is neglected here for simplicity; we caution, however, that the additional presence of fossil electrons in cluster outskirts and in filaments will increase our estimates here, at least limited to $\mathcal{M} \leq 3-4$ shocks in the simulation (while for stronger shocks the direct injection from DSA should dominate the emission in any case). The downstream radio emission is the convolution of the several power-law distributions of electrons that overlap in the cooling region, to which we assign the integrated radio spectrum of $I(v) \propto v^{-s}$, where $s=(p-1) / 2+1 / 2$, with $p=2\left(\mathcal{M}^{2}+1\right) /\left(\mathcal{M}^{2}-1\right)$.

An example of the radio emission at $v=260 \mathrm{MHz}$ from our simulation is given in Fig. 2. The SKA-LOW primary beam at this frequency should be on the order of $\sim 5^{\circ} \times 5^{\circ}$, while our sky model covers $28.4^{\circ} \times 28.4^{\circ}$. The radio emission is clearly more diffuse compared to the X-ray emission, because unlike the latter it does not scale (only) with gas density, but with the shock kinetic power, which can be significant in cluster outskirts (e.g. Ryu et al. 2003). The fact that the radio power typically extends out to larger cluster radii makes it a very good probe of the rarefied cosmic web, but at the same time reduces the chances of overlap with X-ray detections, as we shall see in the next section, with a sweet spot on the scale of cluster outskirts.

\section{Results}

\subsection{X-ray emission and radio emission from the cosmic web}

We started by computing the X-ray emission from the entire simulation, as a function of environment and for different energy ranges, namely [0.3-0.8], [0.8-1.2], [1.2-2.0], [2.0-5.0], and [5.0-7.0] keV, assuming $Z=0.3 Z_{\odot}$ everywhere. In Fig. 3 we show the median and total $X$-ray emission from all pixels in the sky model of Fig. 2, binned as a function of their gas temperature, which is mass-weighted along the entire line of sight ${ }^{4}$.

At $T_{\mathrm{mw}} \leq 5 \times 10^{6} \mathrm{~K}$ (where $T_{\mathrm{mw}}$ is the mass-weighted gas temperature), the X-ray emission from the WHIM is always

\footnotetext{
4 It is important to notice here that the average temperature values along a line of sight of $100 \mathrm{Mpc}$ underestimate by a factor approximately ten (or more) the real temperature values in $3 \mathrm{D}$.
} 


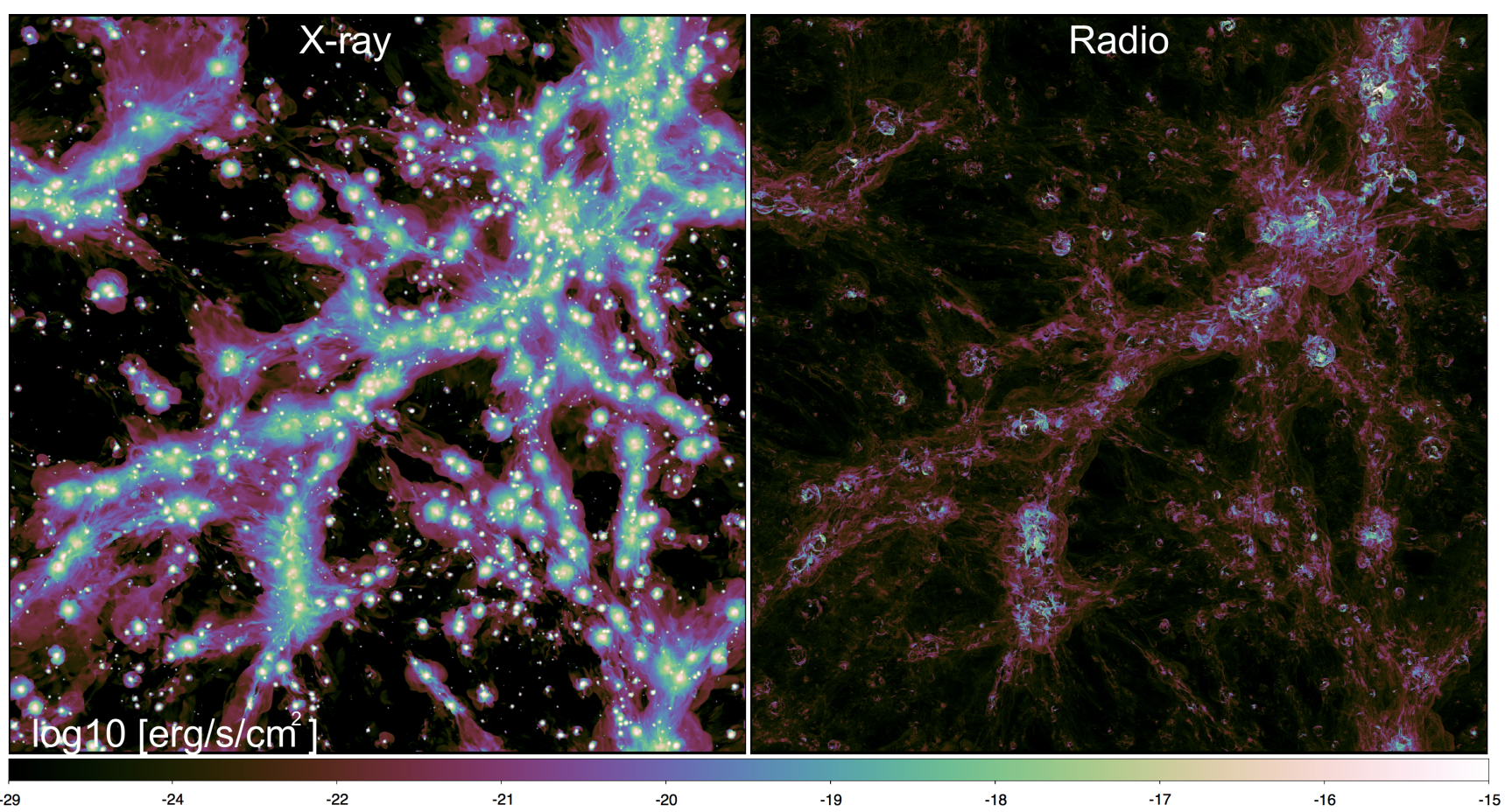

Fig. 2. Projected X-ray emission in the [0.8-1.2] keV band (left) and mock radio emission at $260 \mathrm{MHz}\left(\right.$ right) for our $100^{3} \mathrm{Mpc}^{3}$ volume located at $z \approx 0.05$.

highest in the softest band $(0.3-0.8 \mathrm{keV})$. Most of the emission is free-free radiation above $T_{\mathrm{mw}} \geq 10^{6} \mathrm{~K}$, while at lower temperatures emission lines become dominant. In the entire [0.3-1.2] keV part of the spectrum at these temperatures lines are dominant, and they are approximately ten times more than in the other higher bands (see Sect. 4 for variations in the assumed metallicity of our gas). Conversely, for $T_{\mathrm{mw}} \geq 10^{7} \mathrm{~K}$, the X-ray emission is always more prominent in the $[2.0-5.0] \mathrm{keV}$ band, all contributed by free-free emission.

This suggests that, in principle, the $[0.3-0.8] \mathrm{keV}$ band has the highest chances of detecting the WHIM in emission. However, when the full response of Athena is taken into account, as well as the realistic contribution from astrophysical and instrumental backgrounds, the [0.8-1.2] keV band gives a significantly higher chance of detection. In Sect. 3.3 we will show in detail how our modelling suggests the above finding, which stems from a quantitative comparison of the fraction of pixels tracing various environments across our simulation, which can be detected as a function of exposure time and energy bands for different instruments. While we defer to that section for the demonstration of this finding, we anticipate this result here in order to present more general trends found in our data, limiting our study for simplicity to the [0.8-1.2] keV band in particular.

Second, we characterized the distribution of X-ray and radio emission from the simulated cosmic web, by comparing the $\mathrm{X}$-ray signal in the [0.8-1.2] keV range to the radio emission at $260 \mathrm{MHz}$. In Fig. 4 we show the cumulative and differential distribution of X-ray, $F_{\mathrm{X}}$, and radio flux, $F_{\mathrm{R}}$, for the pixels in Fig. 2, in which no observational cuts are taken into account for now. While both distributions show a similar decreased powerlaw behaviour, the radio distribution has a steeper behaviour for increasing $F_{\mathrm{R}}$, indicating the relative scarcity of powerful detectable radio emission from cluster shocks (e.g. radio relics). Considering that $\sim 10^{-17} \mathrm{erg} \mathrm{s}^{-1} \mathrm{~cm}^{-2}$ is the fiducial value for the future sensitivity of SKA-LOW at this frequency, from this plot we can derive that at most $\sim 1 \%$ of the entire radio emission produced by the cosmic web will be detectable even in the future. Due to the flatter slope of the $F_{\mathrm{X}}$ distribution, for a fiducial detection threshold of $\sim 10^{-16} \mathrm{erg} \mathrm{s}^{-1} \mathrm{~cm}^{-2}$ we have instead that $\sim 10 \%$ of the total thermal emission from the cosmic web in this energy range may be detectable (assuming observations at high galactic latitudes and long exposures). We will comment more on this issue in Sect. 5.

By computing the distribution of $F_{\mathrm{R}}$ as a function of $F_{\mathrm{X}}$ for the pixels in our simulated sky model (Fig. 5), we can demonstrate the lack of a simple relation between the two emission mechanisms. For a given bin in X-ray flux the radio emission can vary by more than six orders of magnitude. Interestingly, we measure that the distribution of $F_{\mathrm{R}}$ in the volume peaks for $10^{-17} \mathrm{erg} \mathrm{s}^{-1} \mathrm{~cm}^{-2} \leq \mathrm{F}_{\mathrm{X}} \leq 10^{-14} \mathrm{erg} \mathrm{s}^{-1} \mathrm{~cm}^{-2}$, that is on the order of the sensitivity level of Athena.

The pixels in this range of $F_{\mathrm{X}}$ are mostly related to the external accretion regions of galaxy clusters and groups, as shown by the different colour-coding adopted in Fig. 5, in which we marked pixels as belonging to clusters or groups in red if they fall within the projected volume $\left(\leq R_{100}\right)$ of halos. Those that are at a projected distance $R_{100} \leq r \leq 3 \cdot R_{100}$ from halos centres are marked in blue. Finally, pixels were marked in green if they belong to filaments, that is, if they have a projected gas density in the range $1 \leq n_{\text {proj }} /\langle n\rangle \leq 50$ and have a projected temperature in the range $10^{5} \mathrm{~K} \leq T_{\mathrm{mw}} \leq 10^{7} \mathrm{~K}$.

As shown by the additional approximate detection limit for future X-ray and radio observations $\left(\sim 10^{-16} \mathrm{erg} \mathrm{s}^{-1} \mathrm{~cm}^{-2}\right.$ for [0.8-1.2] keV and $4 \times 10^{-18} \mathrm{erg} \mathrm{s}^{-1} \mathrm{~cm}^{-2}$ for $260 \mathrm{MHz}$, dashed lines in Fig. 5), for the majority of our simulated pixels $F_{\mathrm{X}} \gg$ $F_{\mathrm{R}}$, that is, the X-ray fluxes are larger than the radio fluxes. The few notable exceptions are the spikes in the radio flux, marked by the vertical stripes in the plot. They are mostly related to temperatures of $T_{\mathrm{mw}} \sim 10^{6}-10^{7} \mathrm{~K}$. A visual inspection shows that such patterns are associated with structure formation shocks, often 

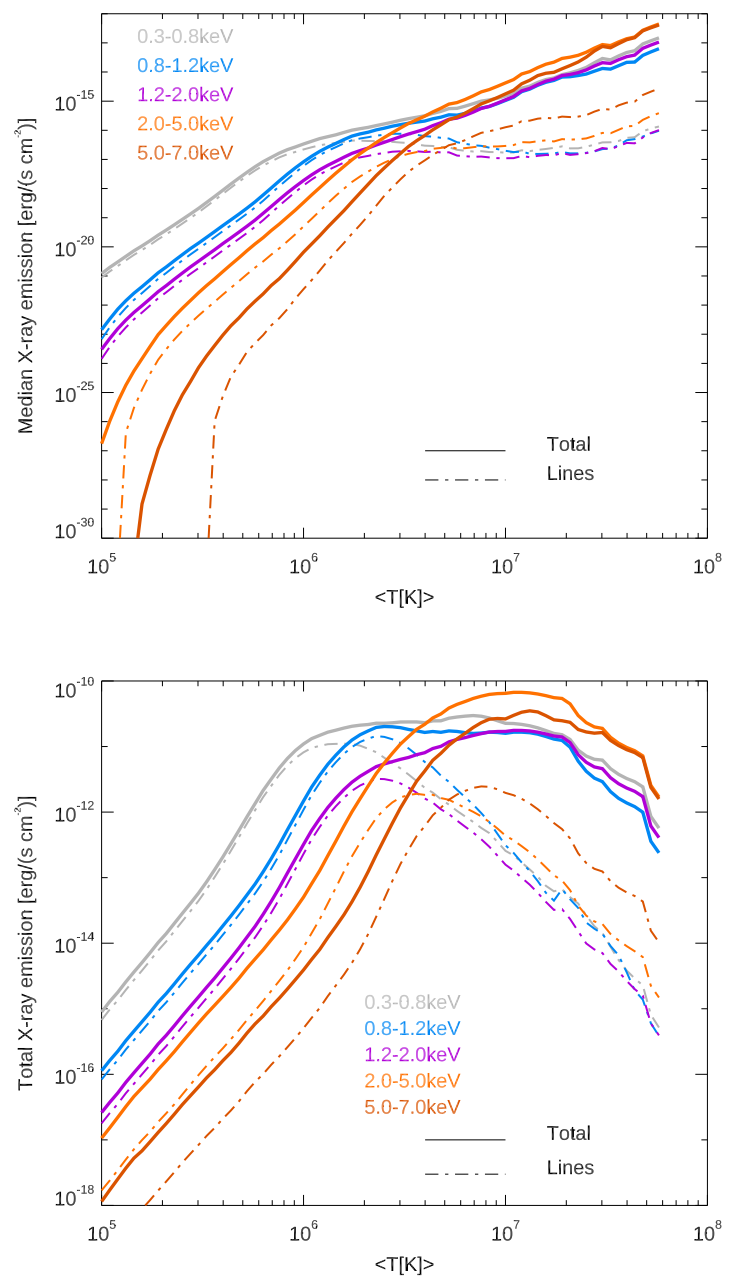

Fig. 3. Distribution function of the median X-ray emission (top) and of the total X-ray emission (bottom) for different energy ranges, as a function of the projected mass-weighted temperature of gas in our simulation. The solid lines give the total X-ray emission, while the dotdashed lines give the contribution only by line emission (assuming a fixed $0.3 Z_{\odot}$ metallicity everywhere).

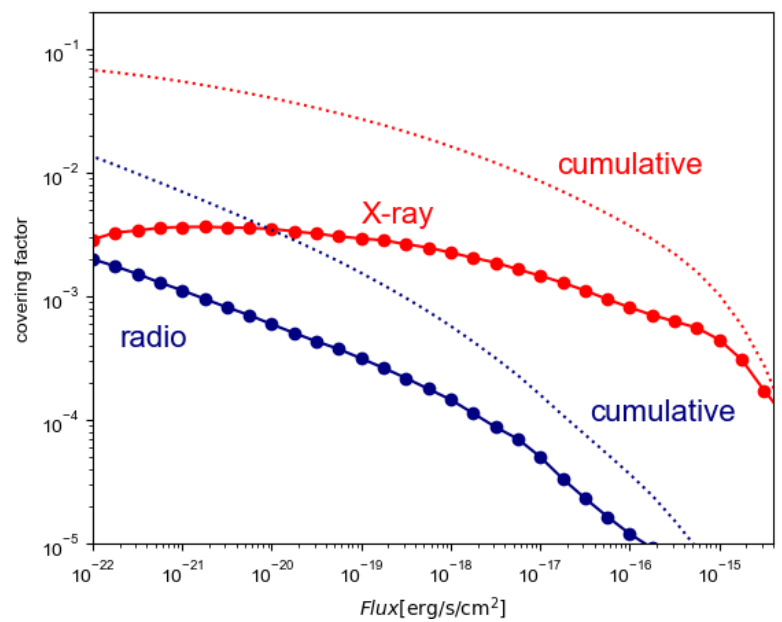

Fig. 4. Area coverage as a function of X-ray and radio flux (in the [0.8-1.2] keV range and at $260 \mathrm{MHz}$, respectively) for the same sky models as Fig. 5. The solid lines give the differential distribution while the dashed lines give the cumulative.

in the periphery of galaxy clusters or between merging cluster pairs, and we will focus on them in the next section.

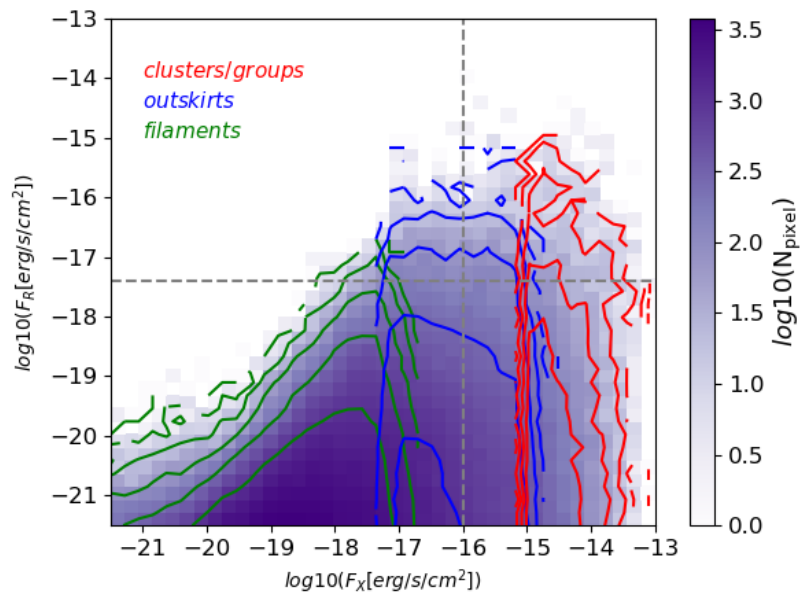

Fig. 5. Distribution of X-ray and radio flux for all simulated pixels in our sky model (Fig. 2). The contours are equally spaced in logarithmic space $\left(\Delta \log _{10}=0.5\right)$ and show the distributions of pixels associated with filaments (green), cluster outskirts (blue), or the virial region of galaxy clusters and groups (red). The additional grey lines delimit the regions that will become observable with future X-ray and radio telescopes.

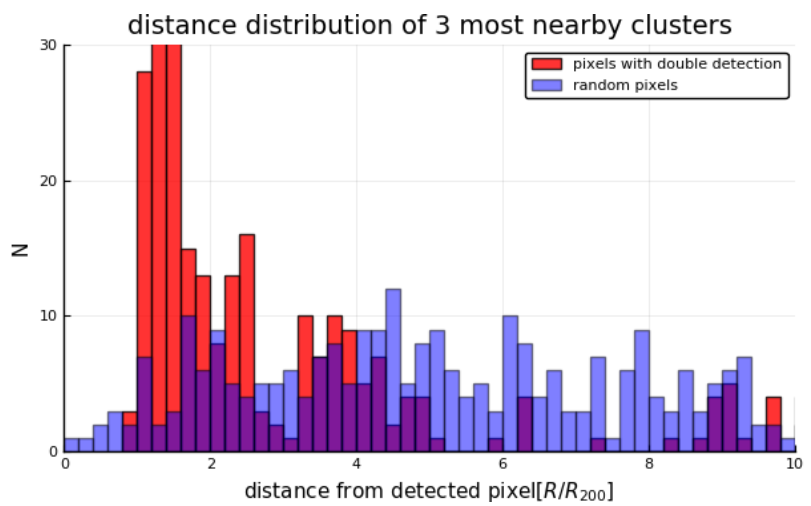

Fig. 6. Distribution of the projected distance from doubly detectable pixels of clusters in our simulation, considering the three most nearby clusters from every pixel. The blue histogram gives the distribution of three most nearby clusters around randomly chosen locations in the simulation.

\subsection{Enhanced gas emission from cluster outskirts}

In this section we wish to identify the most promising targets for a joint detection of the WHIM in the radio and in the X-ray band. To this end, we computed the clustering and morphological properties of massive halos found in the neighbouring pixels that are detected in the X-rays and in radio emission.

Our $100^{3} \mathrm{Mpc}^{3}$ simulation box contains a total of 2347 halos with $M_{100} \geq 10^{12} M_{\odot}, 268$ halos with $M_{100} \geq 10^{13} M_{\odot}$ and 26 halos with $M_{100} \geq 10^{14} M_{\odot}$ at $z=0.05$, consistent with the expected mass function (e.g. Sheth \& Tormen 1999) within the limits of Poissonian statistics. To quantify the association between clusters in the simulation and pixels detected in X-rays and radio, we started by computing the (projected) distance distribution of the three most nearby clusters around each jointly detectable pixel (see Fig. 6). We compared this to a similar distribution drawn for an equal number of randomly drawn positions in the simulation. On average, the pixels that can be detected both in radio and X-rays have a much higher concentration of halos around them. This excess clustering shows up even if we consider the distribution of the ten most nearby clusters around 

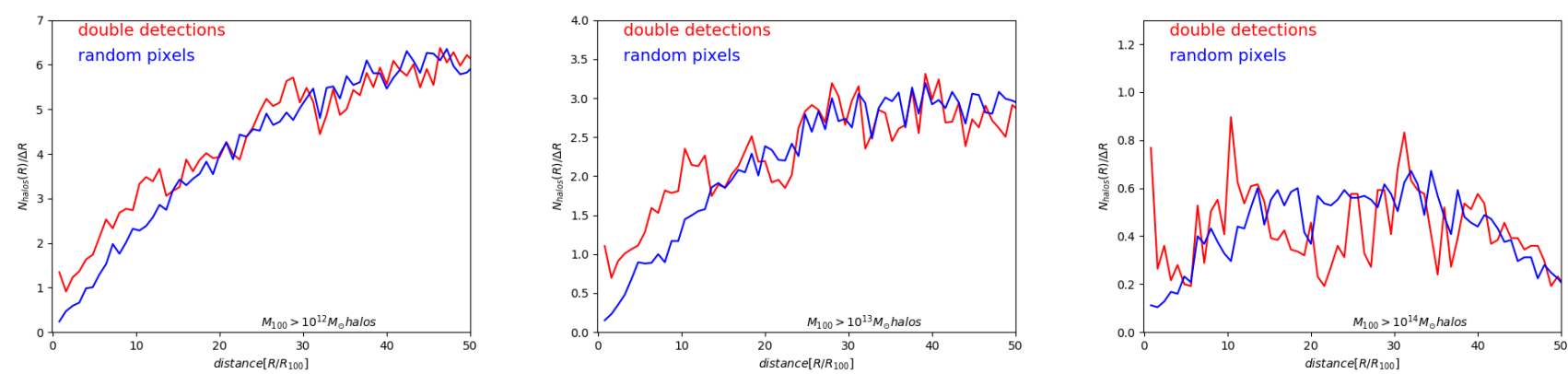

Fig. 7. Average number of clusters (considering a different lower threshold in mass) at a given distance from the location of joint detections in our simulations. The blue data are for the distances computed from a randomly drawn set of positions, for a number of points equal to our jointly detectable pixels.

them. Likewise, the projected radial distribution of the number of halos (considering different mass cuts) from doubly detected pixels shows a significant excess compared to a randomly drawn distribution, up to $\sim 20-30 \mathrm{Mpc}$ distances, as shown in Fig. 7.

In order to investigate clusters in the vicinity of these double detectable regions, we computed the emission of halos at their periphery. In the top panel of Fig. 8 we computed the 95th percentile of X-ray and radio emission at $R_{100}$ from our set of clusters, and plot it as a function of the cluster total mass within the same radius. Large symbols mark the objects in which at least $1 \%$ of the area of the $R_{100}$ shell (considering a width of $41.6 \mathrm{kpc}$ ) can be detected simultaneously in X-rays and in the radio band, given our assumed detection thresholds. At such large distances, the brightest end of the X-ray emission distribution does not scale with cluster mass. The reason is that they are dominated by rare positive brightness enhancements, which are mostly due to the presence of large-scale companions (whose amplitude does not directly correlate with the host cluster mass), while the radio emission is overall well correlated with the host cluster mass.

In our model the radio emission is solely caused by shocks, thus the radio power scales with the shock kinetic power, $\Phi_{\mathrm{K}} \propto$ $\rho v_{\mathrm{s}}^{3} \sim M_{100}^{3 / 2}$. Small departures from this relation are due to projection effects, as well as to the non-linear dependence of the acceleration efficiency on the shock Mach number. In the lower panel of Fig. 8 we quantify the detectability of cluster outskirts for each object, by computing the fraction of a shell that can be observed as a function of the host cluster mass: the odds of a joint detection of cluster outskirts do not scale with the cluster mass, and joint detections are found across the entire mass range of our sample.

We checked whether the enhanced emission in cluster outskirts correlate with large-scale disturbances in the matter distribution, using the mass sparsity simply defined as the ratio between the total mass enclosed within two different radii, for example $S=M_{100} / M_{200}$. While other morphological parameter estimators are useful to characterize the dynamical stage of galaxy clusters in their internal regions, based on X-ray images (e.g. Cassano et al. 2010), the mass sparsity correlates well with cosmological parameters $\left(\sigma_{8}\right.$ and $\left.\Omega_{\mathrm{M}}\right)$ as well as with the dynamical state of clusters to a larger radius (e.g. Corasaniti et al. 2018), and is a convenient parameter to measure as it involves two integrated quantities (e.g. either the total mass or the integrated X-ray luminosity), which should be robustly probed by future X-ray surveys. However, our search for a dependence between the mass sparsity of our cluster sample and enhanced emission in their outskirts did not highlight any dependence as
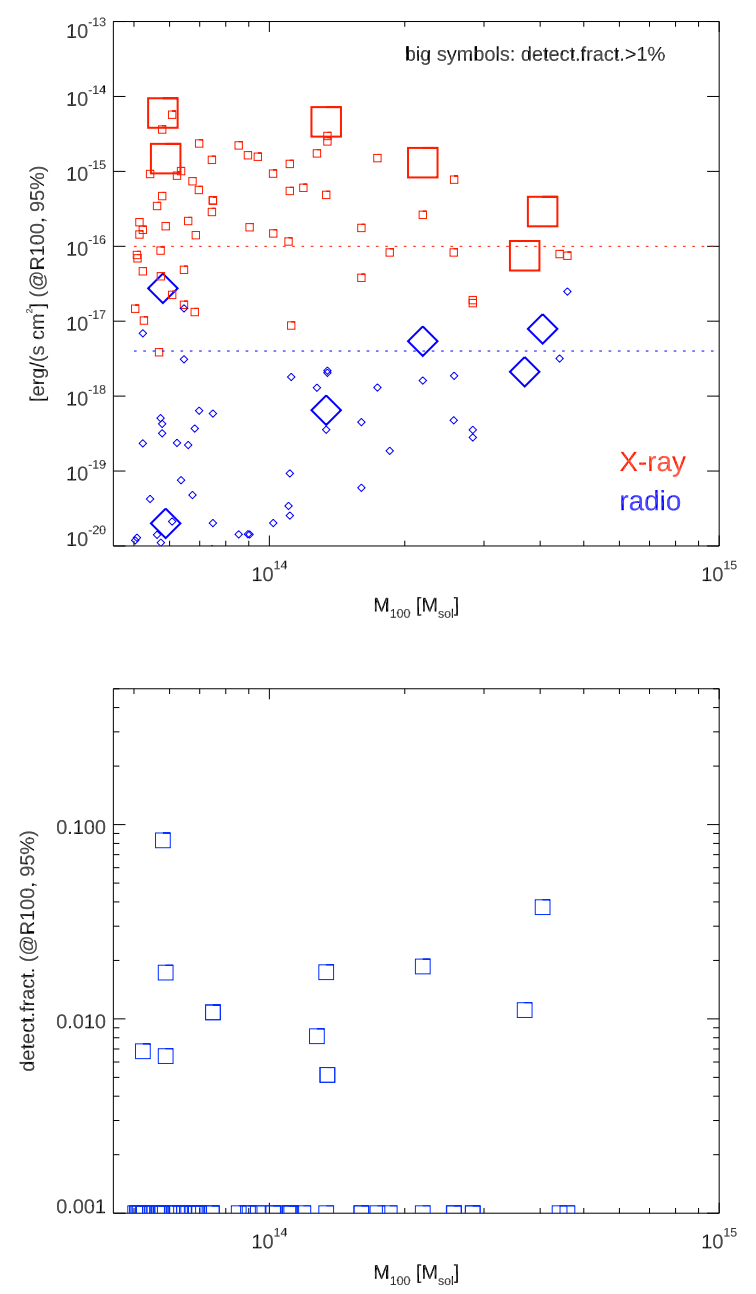

Fig. 8. Top panel: 95 th percentile of the $X$-ray and radio emission from a thin radial shell at $R_{100}$ as a function of the cluster mass, for $\geq 5 \times 10^{13} M_{\odot}$ clusters in our simulation at $z=0.05$. The horizontal lines show the reference detection threshold for both cases. Large symbols correspond to clusters in which $\geq 1 \%$ of the $R_{100}$ shell can be simultaneously detected in X-ray and in radio. Bottom panel: detection fraction for the $R_{100}$ shell in our clusters at $z=0.05$, considering the simultaneous detection in $\mathrm{X}$-ray and radio, as a function of the cluster mass.

doubly detectable regions can be found at both large $(S \sim 1.6)$ and small $(S \sim 1.1)$ values of sparsity.

Next, we analyse the distribution of X-ray and radio emission around our clusters, beginning with the projected radial profile of their emission. In Fig. 9, we show radial distributions of three relevant examples for the average $\mathrm{X}$-ray $([0.8-1.2] \mathrm{keV}$ band) 

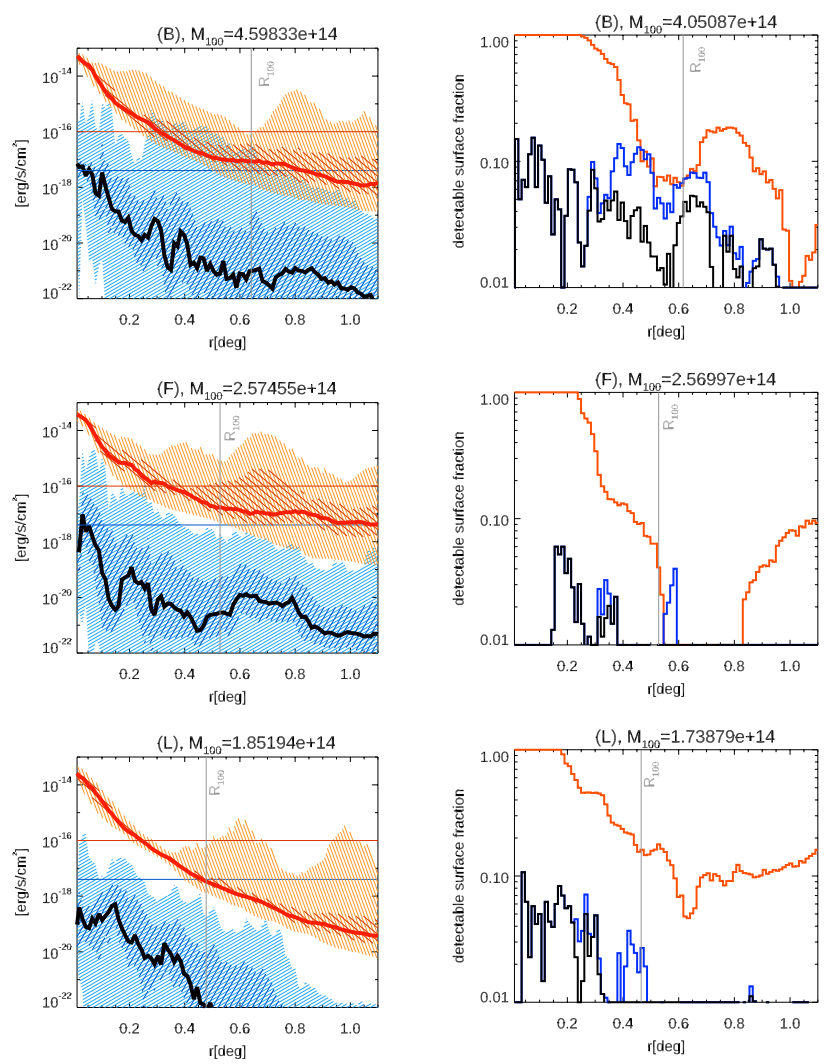

Fig. 9. Left panels: 2D X-ray (in the [0.8-1.2] keV range, in red, and radio emission (at $260 \mathrm{MHz}$, blue curves) profiles for the three halos in our volume at $z=0.05$, showing the median (thick lines) and the $33-66 \%$ and $5-95 \%$ percentile ranges (shaded areas). The horizontal axis gives the angular distance from the centre of each cluster. The horizontal lines give the reference $\mathrm{X}$-ray (red) and the radio (blue) detection threshold considered in the paper, while the vertical grey lines give the location of $R_{100}$ for each cluster. Right panels: radial profiles of the surface fraction that can be detected in X-ray (red), in radio (blue), or by both (black) for the same objects.

and radio $(260 \mathrm{MHz})$ emission in 2D shells around their respective X-ray peaks. For each object, we show the median profiles (thick lines) as well as the 5-25-75-95\% regions around the median. The choice of cutting the profiles for $\geq 95 \%$ limits the contribution from X-ray bright gas clumps, which get typically masked in real observations, and are not good tracers for the ICM and WHIM conditions (e.g. Roncarelli et al. 2006b; Zhuravleva et al. 2011; Eckert et al. 2013). The surface fraction that can be detected in the X-ray or in the radio domain as a function of the angular distance from the cluster centre of the 20 most massive clusters in our sample is given in the right panels of the same figure. For each cluster, the vertical axis gives the fraction of the surface that is above the detection threshold in X-ray, in radio, or simultaneously in both.

In roughly half of our sample a significant level of the $\mathrm{X}$-ray emission should be detectable by Athena at the projected $R_{100}$ (consistent with Fig. 8), which typically corresponds to $\sim 0.4-0.6^{\circ}$ from the cluster centre. Conversely, in the radio domain several clusters host detectable radio emission in their innermost regions, which is associated to transient powerful merger shocks, leading to "classic" radio relics (van Weeren et al. 2019). Even with the planned sensitivity of SKA-LOW at $260 \mathrm{MHz}$, the average radio emission there will be well below the detection threshold in most cases, and only in $\sim 10-20 \%$ of clusters do we expect direct single detection of the shocked

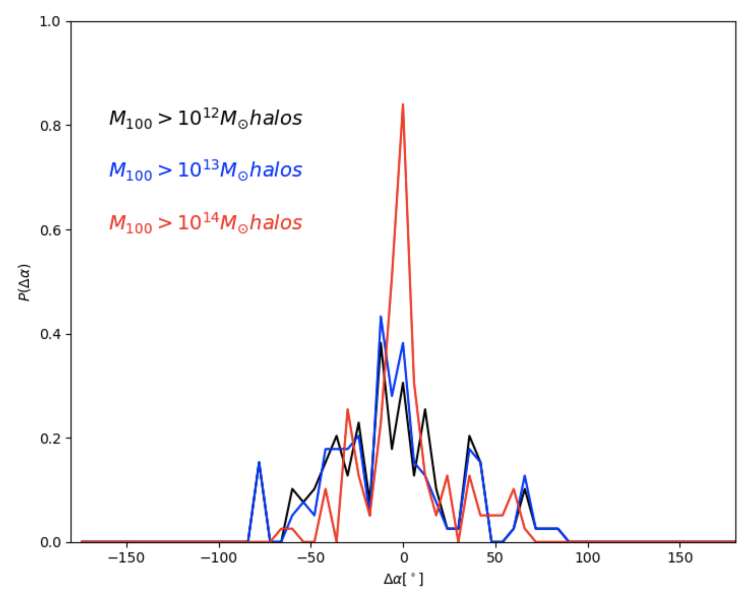

Fig. 10. Distribution of position angle between double detectable pixels at $z=0.05$ and the two closest galaxy clusters to each of them (see text for more explanations). The different colours refer to different cuts in the cluster mass within our sample.

WHIM at the virial radius ${ }^{5}$, while a detection is guaranteed via stacking techniques (Vazza et al. 2017a).

The second and third clusters shown in Fig. 9 (identified as "F" and "L" in the panels) are the most frequent cases: there a non-negligible fraction (5-20\%) of the $R_{100}$ shell is detectable in $\mathrm{X}$-rays with Athena, while in both cases only a very tiny fraction of radio emission would be detectable by SKA-LOW. Moreover, there is no spatial overlap between X-ray and radio detection, hence Athena and SKA will not be able to study the same portion of the ICM and WHIM in these objects, which are the majority in our sample.

However, in the first system ("B" in Fig. 9) $~ 10 \%$ of the $R_{100}$ shell will be detectable in X-ray with Athena, as well as $\sim 5-10 \%$ in the radio domain, with a non-negligible overlap of detectable regions in the two instruments, offering the chance joint analysis. A striking difference emerges from the X-ray profile, and may offer the key to predict the occurrence of such rare configurations: there is a prominent secondary X-ray emission peak outside of the virial radius of the first cluster, indicating the presence of a massive nearby cluster companion. This is not present in the profile of cluster "L", while a secondary peak is seen in cluster " $F$ " but at a much larger distance from the virial radius of the main cluster.

Systems such as "B" only account for $\sim 10-20 \%$ of our sample, and they suggest that the presence of a massive companion, likely in an early interaction stage, is key to producing X-ray detectable bridges that are engulfed by detectable radio emission. How general is this finding?

We further explored the link between joint X-ray and radio detections and merging clusters by studying the distribution of relative position angles between cluster pairs found around doubly detectable pixels in our sky model. In Fig. 10, we show the distribution of $\Delta \alpha$, the angle between the projected lines connecting the centres of the two most massive clusters (considering different mass cuts) around doubly detectable pixels. If the two lines connecting the centres of each cluster are perfectly aligned, $\Delta \alpha=0^{\circ}$, while large values of $|\Delta \alpha|$ indicate a misalignment between detectable pixels and surrounding pairs of clusters. The

\footnotetext{
5 We notice that here our emission model is more conservative with respect to our previous analysis in Vazza et al. (2017a), in which a ten times larger initial seed field was assumed.
} 


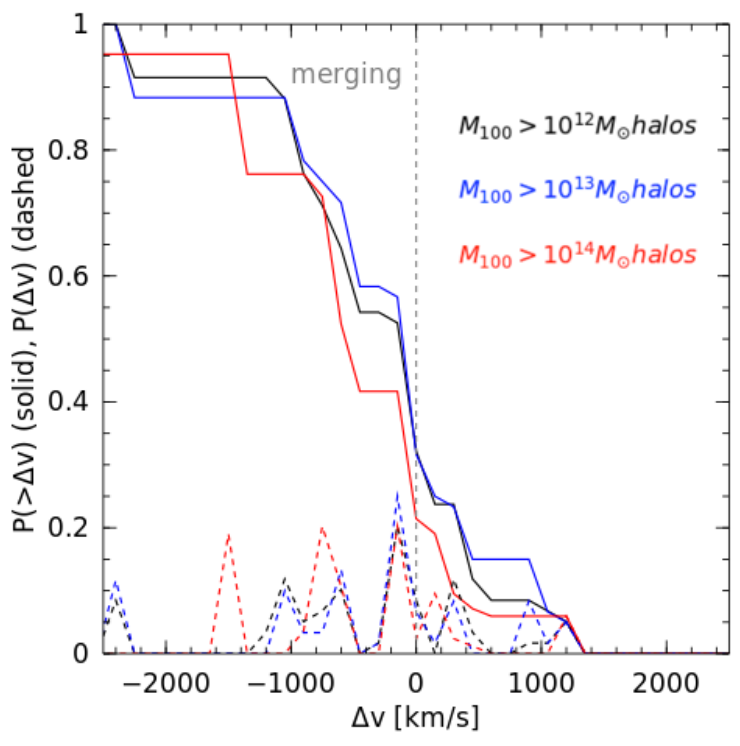

Fig. 11. Cumulative (solid) and differential (dashed) distributions of the relative velocities between the two closest galaxy clusters around all doubly detectable pixels in our run at $z=0.05$, limited to objects with a position angle $|\Delta \alpha| \leq 25^{\circ}$ in Fig. 10. The different colours refer to different cuts in the cluster mass within our sample.

distribution of angles peaks at $\Delta \alpha \sim 0^{\circ}$ and most cluster pairs (especially when $\geq 10^{14} M_{\odot}$ ) have $|\Delta \alpha| \leq 25^{\circ}$.

We can also measure the relative gas velocities (measured as the median in the core cluster region) between clusters with $|\Delta \alpha| \leq 25^{\circ}$, for the same mass selections. This is shown in Fig. 11, where we computed the (cumulative and differential) distributions of these relative velocities. Especially when clusters with masses larger than $10^{14} M_{\odot}$ are considered, in $\sim 80 \%$ of cases the relative velocities between the cluster cores are significantly negative, $\Delta v \sim-(1000 \div 3000) \mathrm{km} \mathrm{s}^{-1}$. This confirms that most of such systems are colliding, approximately along the straight line connecting their cores, where also most of our doubly detectable pixels are located.

Such cluster-cluster bridges are remnants of large-scale filaments once connecting these systems. By the time at which interacting systems have nearly intersecting virial regions, the gas flow between them reaches $\sim 1000-2000 \mathrm{~km} \mathrm{~s}^{-1}$ velocities, and this forces their outer gas layers to be significantly compressed. This compression causes a boost of the X-ray emission level, roughly on the order of $\sim\left(\rho_{2} / \rho_{1}\right)^{11 / 4}$ if $\rho_{2}$ is the gas density after the compression and $\rho_{1}$ was the gas density before the close encounter ${ }^{6}$. Moreover, the increase in gas temperature boosts the chance of detecting this tenuous gas phase in the [0.8-1.2] keV, by letting a larger portion of the X-ray emission spectra "enter" the soft X-ray window. On the other hand, the implied shock velocities are modest, with typical Mach numbers of $\mathcal{M} \leq 5-10$, fragmented on $\sim 10^{2} \mathrm{kpc}$ scales. We will analyse in detail one such system at higher spatial resolution in Sect. 3.3.4.

Such double regions are parts of the WHIM that resemble the periphery of clusters. As already noticed by other authors (e.g. Roncarelli et al. 2006b; Iapichino et al. 2011) the usage of a too rigid temperature selection to define the WHIM phase, for example $T \sim 10^{5}-10^{7} \mathrm{~K}$, does not fully capture the different possible histories of the weakly X-ray emitting gas that may be detected in the periphery of galaxy clusters.

6 This follows from considering $L_{\mathrm{X}} \propto \rho^{2} \sqrt{T}$ and $T \propto \rho^{3 / 2}$ in the adiabatic case.
Table 1. Values adopted for our mock X-ray observations with Athena, eROSITA, and XMM-Newton.

\begin{tabular}{lcccc}
\hline \hline Instrument & $\begin{array}{c}\text { Energy band } \\
(\mathrm{keV})\end{array}$ & $\begin{array}{c}B_{\mathrm{bg}} \\
\left(\frac{\mathrm{counts}}{\text { arcmin }^{2} \mathrm{Ms}}\right)\end{array}$ & $f_{\mathrm{abs}}$ & $\begin{array}{c}A_{\mathrm{eff}} \\
\left(\mathrm{cm}^{2}\right)\end{array}$ \\
\hline Athena-WFI & $0.3-0.8$ & $2.1 \times 10^{4}$ & 0.83 & 9511 \\
& $0.8-1.2$ & $3.1 \times 10^{3}$ & 0.95 & 12139 \\
& $1.2-2.0$ & $1.4 \times 10^{3}$ & 0.98 & 10841 \\
& $2.0-5.0$ & $3.4 \times 10^{3}$ & 0.99 & 4673 \\
& $5.0-7.0$ & $2.0 \times 10^{3}$ & 1.00 & 2131 \\
\hline eROSITA & $0.3-0.8$ & $2.2 \times 10^{3}$ & 0.83 & 610 \\
& $0.8-1.2$ & $4.6 \times 10^{2}$ & 0.95 & 1243 \\
& $1.2-2.0$ & $4.2 \times 10^{2}$ & 0.98 & 1267 \\
& $2.0-5.0$ & $4.1 \times 10^{2}$ & 0.99 & 287 \\
XMM & $5.0-7.0$ & $3.0 \times 10^{2}$ & 1.00 & 88 \\
\hline PN + 2MOS $)$ & $0.3-0.8$ & $4.6 \times 10^{3}$ & 0.83 & 1056 \\
& $0.8-1.2$ & $1.4 \times 10^{3}$ & 0.95 & 1655 \\
& $1.2-2.0$ & $1.8 \times 10^{3}$ & 0.98 & 1894 \\
& $2.0-5.0$ & $3.4 \times 10^{3}$ & 0.99 & 1337 \\
& $5.0-7.0$ & $1.7 \times 10^{3}$ & 1.00 & 998 \\
\hline
\end{tabular}

Notes. For each different energy band we give the count rate due to the effective (sky+instrumental) background, the fraction $f_{\text {abs }}$ of source counts unabsorbed by the galactic column density (assuming $n_{\mathrm{H}}=$ $2 \times 10^{20} \mathrm{~cm}^{-2}$ ), and the mean effective collecting area $A_{\text {eff }}$ in that energy range. For eROSITA, we quote values extrapolated from http://www.mpe.mpg.de/1303215/eROSITA_background_v12. pdf (Boller, priv. comm.); for XMM-Newton, we quote an average value on a set of observations obtained under different conditions (of orbits, solar cycle, time; Ghirardini, priv. comm.).

\subsection{Synthetic observations}

Here we discuss strategies to detect the gas emission located in bridges of close cluster pairs. In order to quantify the detectability of intracluster gas bridges with existing or future observations, we produced a small survey of mock observations in X-ray and radio bands, assuming either the specifications for wide-area surveys of specific instruments, as well as the specifications for dedicated reprocessing (e.g. with UV tapering in the radio case) of specific objects in order to increase the sensitivity to diffuse emission.

\subsubsection{X-ray: Athena, eROSITA, and XMM}

Here we compare the performances of shallow full-sky surveys with eROSITA (either $1.6 \mathrm{ks}$ in the entire sky or $20 \mathrm{ks}$ in the polar regions), with long targeted exposures with Athena's Wide-Field Imager (1 Ms or $100 \mathrm{ks}$ ) and with XMM-Newton (100 ks), for different energy bands. To quantify the observable regions of each mock observation we use the signal-to-noise ratio $(\mathrm{S} / \mathrm{N})$, quantified as

$S / N=\frac{f_{\mathrm{abs}} \cdot S}{\sqrt{f_{\mathrm{abs}}\left(S+2 B_{\mathrm{bg}}\right)}}$,

where $S$ is the number of photon counts originating from the source within a given energy band and collected by the effective area $A_{\text {eff }}, f_{\text {abs }}$ is the ratio between the absorbed (from an assumed galactic column density of $n_{\mathrm{H}}=2 \times 10^{20} \mathrm{~cm}^{-2}$ ) and not absorbed source counts for a typical plasma with gas temperature of $3 \mathrm{keV}$, metallicity of 0.3 times the solar value, and $B_{\mathrm{bg}}$ is the estimated total X-ray background, including the contributions 

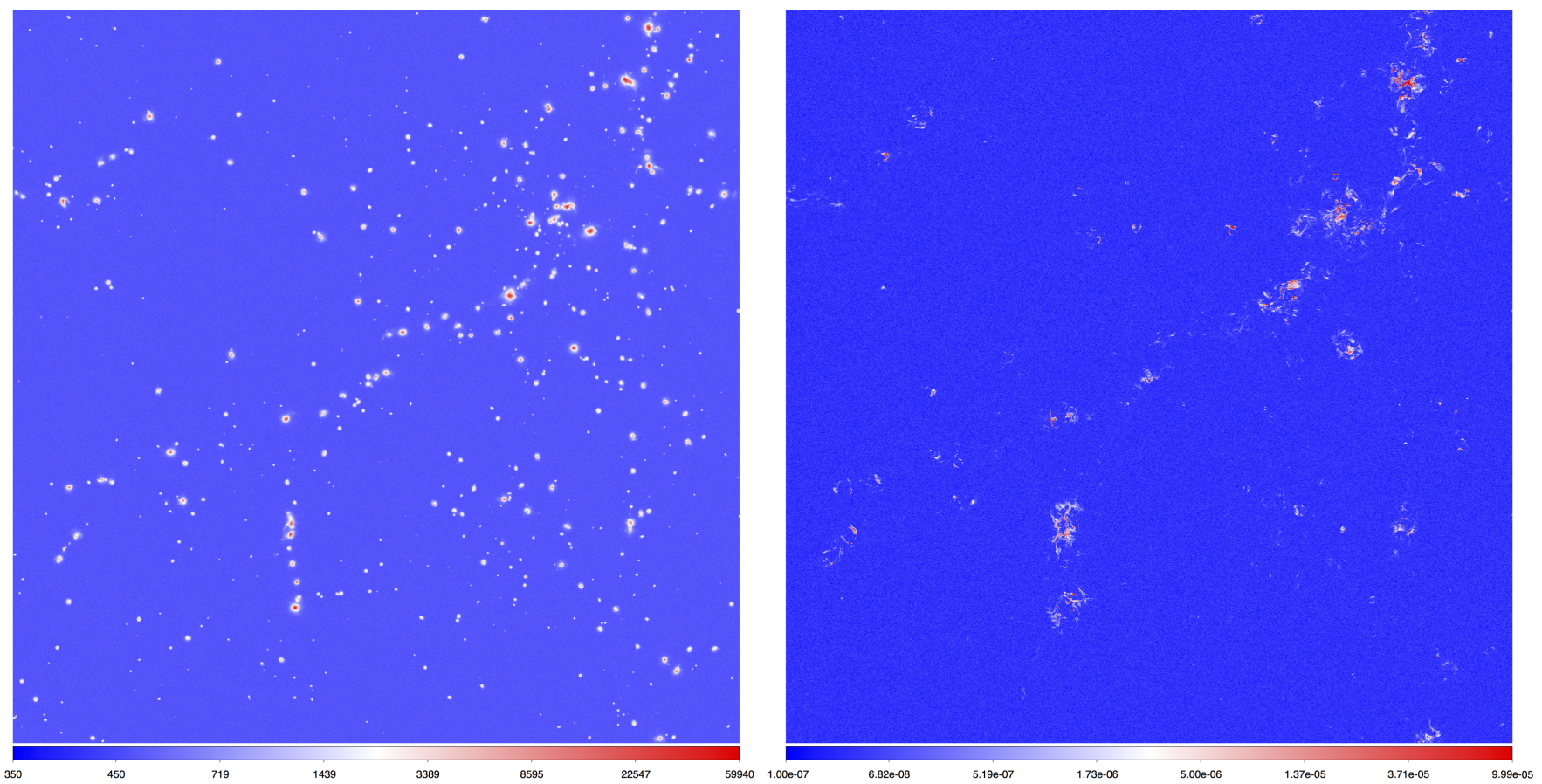

Fig. 12. Mock X-ray (left) and radio (right) observations of the same simulated box of Fig. 1, located at $z \approx 0.05$. The left panel shows the photon counts for a $1 \mathrm{Ms}$ integration in the [0.8-1.2] keV band using WFI (see Sect. 2.2.1 for details) while the right panel gives the result of a mock radio survey at $260 \mathrm{MHz}$ with the SKA-LOW (see Sect. 2.2.2 for details), in units of [Jy arcsec ${ }^{-2}$ ]. Each panel covers $\sim 28.4^{\circ} \times 28.4^{\circ}$ in the sky. Both images include the noise level expected for the respective instrument, band, and integration time (in the case of SKA-LOW, we also consider the confusion noise level).

from the Milky Way, the unresolved (20\% of the total) cosmic X-ray background and the instrumental background ${ }^{7}$. The details of the mock observing parameters considered here are given in Table 1.

The left panels of Figs. 12 and 13 show the mock X-ray exposure maps of our simulated $100^{3} \mathrm{Mpc}^{3}$ located at $z=0.05$ in the [0.8-1.2] keV band, for a $1 \mathrm{Ms}$ exposure in every pixel of the box and considering background counts as in Table 1 for an AthenaWFI observation. The yellow rectangles in Fig. 13 show that extended tails of detectable X-ray emission can often be seen between closely interacting systems (only a fraction of which corresponds to physically bound objects actually interacting in three dimensions).

To quantify the fraction of the gas in the cosmic web that can be detected by each instrument in different bands, it is convenient to measure the typical $\mathrm{S} / \mathrm{N}$ that each observing strategy can achieve as a function of the local temperature, which we can parametrize through the mass-weighted mean temperature along the line of sight within each pixel.

Figure 14 displays the median and the maximum S/N for pixels in different environments and for different energy ranges in Athena's WFI integration of $1 \mathrm{Ms}$. The lower panel in the same figure shows the detectable fraction of the sky model (marked as $S / N \geq 3$ ) as a function of temperature. As anticipated in Sect. 3.1, the combination of the instrumental and particle background, of the galactic absorption, and of the effective collecting area as a function of energy makes the [0.8-1.2] keV slightly

\footnotetext{
7 We use the responses available for each telescope at the following addresses: https://www - the-athena-x-ray-observatory. eu/resources/simulation-tools.html (for Athena), http:// www2011.mpe.mpg.de/erosita/response/(eROSITA), https:// www . cosmos.esa.int/web/xmm-newton/epic-response-files (XMM-Newton).
}

better compared to the [0.3-0.8] keV range for a detection of the emission from the baryons in the (projected) gas temperature range $T \geq 10^{6}-10^{7} \mathrm{~K}$, which are more connected to cluster outskirts and cluster-cluster bridges. In this range, we expect that $\sim 40 \%(\sim 80 \%)$ of the gas with a projected gas temperature of $\sim 10^{6} \mathrm{~K}\left(\sim 5 \times 10^{6} \mathrm{~K}\right)$ can be detected with high significance with a $1 \mathrm{Ms}$ integration, respectively. The detection fraction drops to $\sim 20 \%$ at $T \sim 10^{6} \mathrm{~K}$ in the [2.0-5.0] and to $\leq 1 \%$ in the [5.07.0] keV range, respectively.

In Fig. 15 we compare instead the distribution of the detectable fraction of the sky model for Athena, eROSITA, and XMM, for hypothetical $10 \mathrm{ks}$ (dot-dash lines), $100 \mathrm{ks}$ (dashed), or $1 \mathrm{Ms}$ (solid) exposures. The better performances expected from Athena across the entire energy range stem for the large collecting area in each energy bin. Owing to their smaller $A_{\mathrm{eff}}$, for the same exposures XMM and eROSITA yield significantly lower (factor of approximately two to three) detection fraction for $\leq 10^{7} \mathrm{~K}$ in the $[0.3-0.8] \mathrm{keV}$ and $[0.8-1.2] \mathrm{keV}$ bands, while their performances drop more $(\sim 10)$ in the $\geq 1.2 \mathrm{keV}$ energy range. Below $1.2 \mathrm{keV}$, our statistics suggest that $\sim 1 \mathrm{Ms}$ integrations with XMM and eROSITA would be competitive with a $\sim 100 \mathrm{ks}$ integration with ATHENA. However, in practice eROSITA is designed to perform shallower all-sky surveys (e.g. Borm et al. 2014), integrating for $\sim 1.6 \mathrm{ks}$ in most of the sky, and up to $\sim 20 \mathrm{ks}$ for the regions around the poles. On the other hand, $\sim 50 \mathrm{ks}$ exposures with XMM have been performed in the outer regions of galaxy clusters, leading for example to the detection of intracluster filaments in A2774 (e.g. Eckert et al. 2015).

\subsubsection{Radio: SKA-LOW and LOFAR}

We also produced mock radio observations at a given frequency $v$, for the all-sky survey planned for MWA, LOFAR-HBA, and 

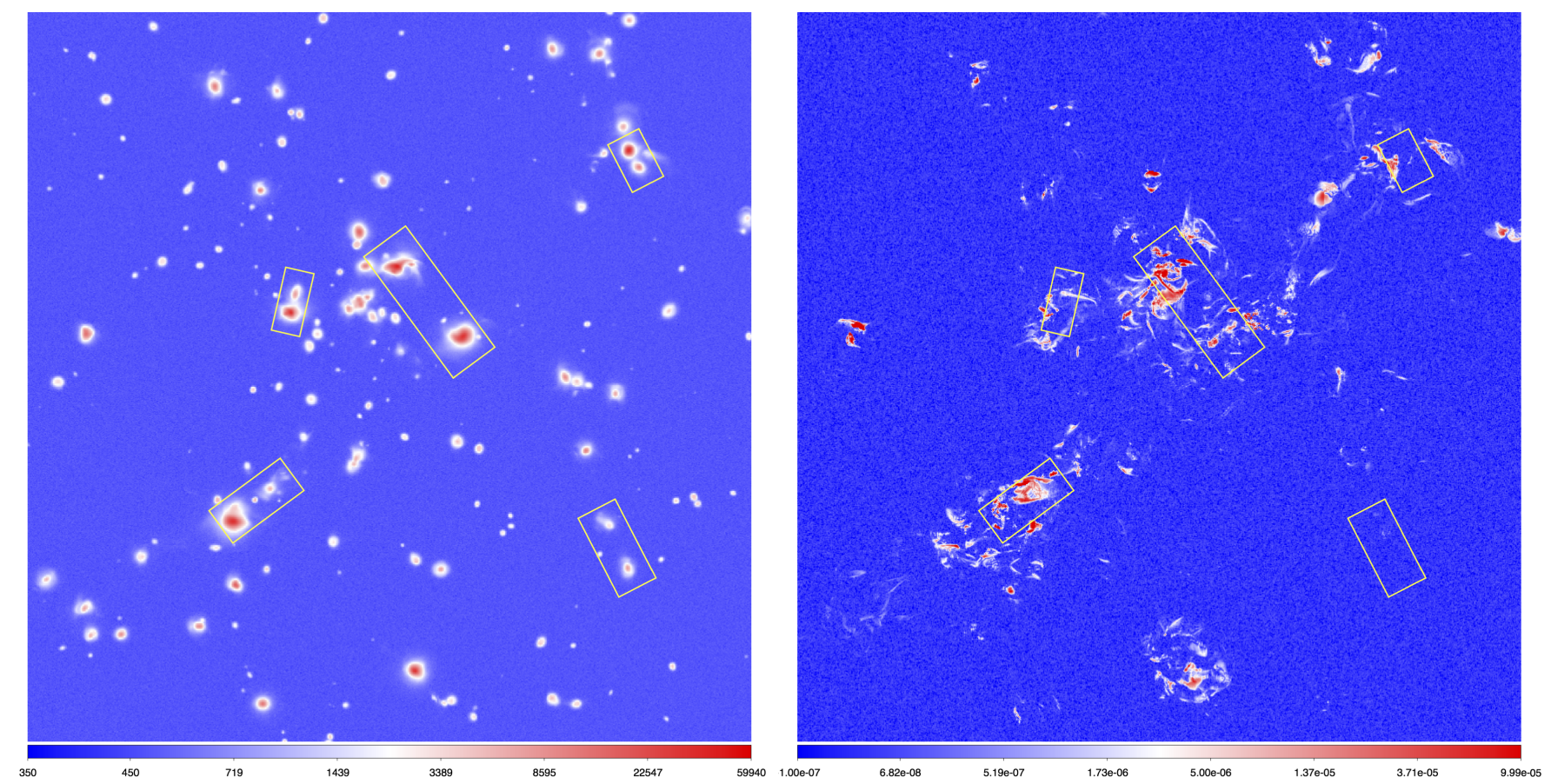

Fig. 13. Close-up view of a crowded $\sim 30 \times 30 \mathrm{Mpc}^{2}$ region of Fig. 2 . Each panel covers $\sim 8.5^{\circ} \times 8.5^{\circ}$ in the sky. As in Fig. 12 , the left panel gives the photon counts for a $1 \mathrm{Ms}$ integration in the [0.8-1.2] keV band using WFI while the right panel gives the result of a mock radio survey at $260 \mathrm{MHz}$ with the SKA-LOW, in both cases with noise included. The additional yellow rectangles show pairs of clusters with ongoing mergers.

SKA-LOW with a procedure similar to Vazza et al. (2015a,b). The radio sky models were transformed using fast Fourier transform to remove the frequencies below the minimum antenna baseline of each specific radio configuration, that is we mimic the loss of signal from scales larger than those sampled by the minimum instrumental baseline. This is particularly relevant for the large-scale diffuse gas emission from filaments and cluster outskirts, even if all these low-frequency telescopes are suitable to effectively sample such large-scale fluctuations, as well as telescopes working at $\sim 1.4 \mathrm{GHz}$. Our maps are converted back into real space and the emission is convolved for the resolution beam with a Gaussian filter, and the detectable emission is only that $\geq 3 \sigma_{\text {rms }}$ (where $\sigma_{\text {rms }}$ is largest between the thermal or the confusion noise of each instrument). In this simplistic approach we assume that it is possible to entirely remove galactic foregrounds (e.g. Bonaldi \& Brown 2015) and point-like radio sources (e.g. van Weeren et al. 2016), so that the theoretical thermal and confusion noise (e.g. Loi et al. 2019) of each instrument can be reached.

The details of the mock observing parameters considered in this work are given in Table 2, in which we compared the performances of our reference SKA-LOW $260 \mathrm{MHz}$ observation to the lower $120 \mathrm{MHz}$ central frequency of LOFAR-HBA and to a $200 \mathrm{MHz}$ observation with MWA Phase I. The left panels of Figs. 12 and 13 show the mock radio observation of our $100^{3} \mathrm{Mpc}^{3}$ box at $z=0.05$ at the central observing frequency of $260 \mathrm{MHz}$, assuming the resolution, baseline sampling, and sensitivity of the SKA-LOW observing conditions given in Table 2. The typical trends of detections we can expect from these instruments applied to the quest for the cosmic web have been already discussed in depth in previous work (e.g. Vazza et al. 2015b, 2017a) and are applicable also here, albeit for SKALOW we consider the most updated predictions on the survey performance.
While our main focus here is to assess the potential of each all-sky radio survey in detecting promising candidates for longer $\mathrm{X}$-ray integrations outside of the virial region of clusters, the peak performance on diffuse emission that each radio telescope can achieve is slightly larger, that is, by observing the same target for longer exposures and/or tapering the data to a coarser resolution to increase the surface brightness sensitivity, if confusion noise is not the limiting factor. We comment on this issue more broadly in the next section. In general, the conclusions reached in our previous works (Vazza et al. 2015a,b, 2017a) apply also here: while the SKA-LOW promises to detect with higher fidelity a non-negligible fraction of the radio emitting cosmic web, both LOFAR and MWA should be able to perform earlier detections of cluster outskirts and emission in-between interacting clusters.

\subsubsection{What can be detected with realistic observations?}

Simulations can help us in defining the most efficient strategy to select targets for long spectroscopic analysis of cluster bridges. Based on our catalogue of halos, we extracted all possible close cluster pairs, with the priors that their virial spheres (based on $R_{100}$ ) should not overlap, and that their projected separation is smaller than four times $R_{12}=R_{100,1}+R_{100,2}$. We then selected a rectangle along the line connecting their centres, starting from one virial radius and ending at the other, with a $5^{\prime}$ width, which corresponds to Athena's X-IFU field of view.

An example is shown in Fig. 16, where we show mock radio and X-ray observations of two bridges connected to a $\sim 2 \times 10^{14} M_{\odot}$ galaxy cluster. Contours indicate the regions that can be detected at $\geq 3 \sigma$ in the different frequencies.

All radio surveys should be able to detect emission from shocked gas in the larger bridge in the centre of the image, while only SKA-LOW should detect some small emission patches in the smaller bridge. While the sensitivity in surface brightness can be increased by changing the observing strategy, for 

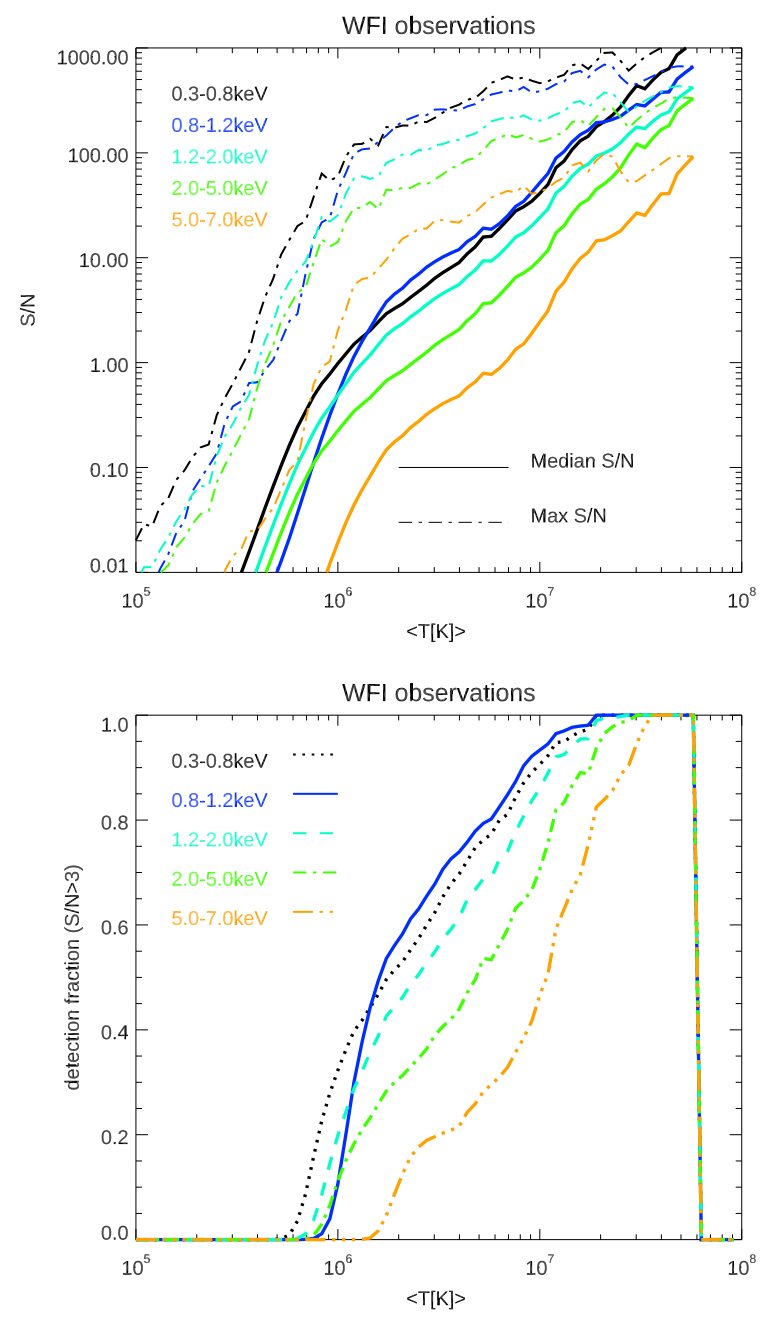

Fig. 14. Top panel: median and maximum $\mathrm{S} / \mathrm{N}$ of pixels in our simulated WFI observation, assuming a $1 \mathrm{Ms}$ integration, as a function of environment and for different energy ranges. Bottom panel: fraction of pixels with $S / N \geq 3$ as function of temperature and energy bands for the same mock Athena-WFI observations.

example by ad-hoc tapering (e.g. Botteon et al. 2018), the high spatial resolution of SKA-LOW $\left(\theta=7.3^{\prime \prime}\right)$ is key to resolving the intricate shock network in bridges. In the same figure we also show that, unlike in the case of large-scale diffuse emission expected from cosmic filaments, the relatively small-scale $\left(\leq 5-10^{\prime}\right)$ emission from shocks in bridges is also well sampled at higher frequencies. In this case, observations at $1.4 \mathrm{GHz}$ with ASKAP or SKA-MID could detect this emission. Moreover, the possibility of observing polarized emission from such regions will enable SKA-MID to make more significant detections since polarization reduces the dynamic range and the confusion level, which is the biggest constraint for SKA-LOW (e.g. Vazza et al. 2015a)

The lower row shows the performance of X-ray observations, considering a $1 \mathrm{Ms}$ Athena-WFI integration of the same region, $100 \mathrm{ks}$ with XMM or $1.6 \mathrm{ks}$ as in the eROSITA all-sky survey. Clearly, the detections of such regions in X-rays will remain a challenge, in which only Athena promises some joint detection with radio observations ${ }^{8}$.

\footnotetext{
8 Since the resolution of our simulation is coarser than the equivalent spatial resolution of these instruments at this redshift, the capabilities of Athena in resolving small-scale structures in the ICM volume are underestimated here.
}
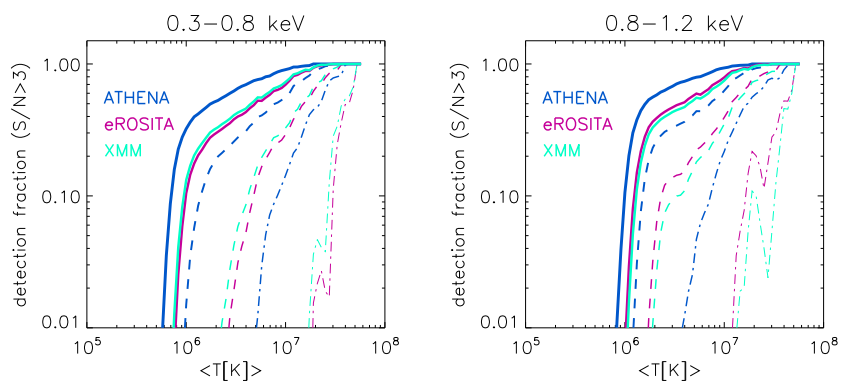

$.2-2.0 \mathrm{keV}$
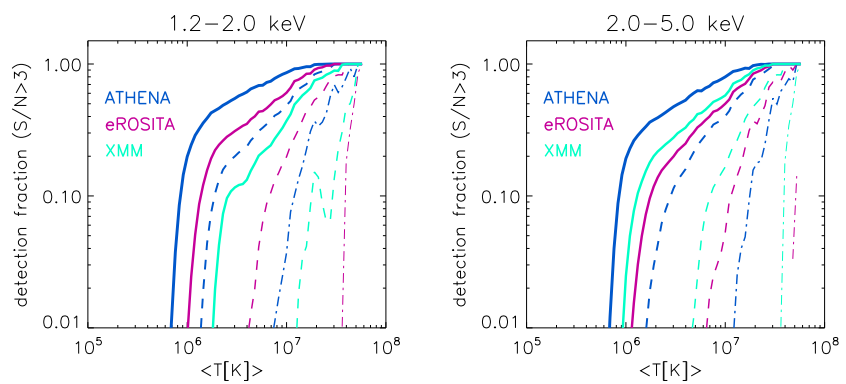

Fig. 15. Distribution of the detectable fraction $(S / N \geq 3)$ of the simulated cosmic web as a function of the projected mass-weighted gas temperature, for the different X-ray satellites considered in this work, for different energy ranges, and considering a $10 \mathrm{ks}$ (dot-dashed lines), a $100 \mathrm{ks}$ (dashed), and $1 \mathrm{Ms}$ (solid) integration.

Table 2. Assumed values for the radio observing parameters considered in this work: central observing frequency, beam resolution, thermal rms noise per beam, and detection threshold considered in our analysis (considering a $3 \sigma$ detection, also including confusion noise).

\begin{tabular}{lcccc}
\hline \hline Telescope & $\begin{array}{c}\text { Frequency } \\
(\mathrm{MHz})\end{array}$ & $\begin{array}{c}\text { Beam } \\
\left({ }^{\prime \prime}\right)\end{array}$ & $\begin{array}{c}\sigma_{\text {rms }} \\
\left(\mu \mathrm{Jy} \mathrm{beam}^{-1}\right)\end{array}$ & $\begin{array}{c}\text { Detection thr. } \\
\left(\mu \mathrm{Jy} \mathrm{arcsec}^{-2}\right)\end{array}$ \\
\hline SKA-LOW & 260 & 7.3 & 4.8 & 0.24 \\
LOFAR-HBA & 120 & 25 & 250 & 1.05 \\
MWA Phase I & 200 & 120 & 10000 & 1.83 \\
\hline
\end{tabular}

In order to quantify the performance of real observations of intracluster bridges, we extracted from our catalogue of pairs the fraction of the connecting rectangle $\left(5^{\prime}\right.$ wide) that can be significantly detected with various instruments and observing techniques. We then averaged the results by rescaling by the distance between the two virial spheres. With a few variations in the selection criteria and in the observing techniques, we find:

- Performance of X-ray versus radio observations: in Fig. 17 we show the mean detectable fraction of intracluster bridges as a function of the distance from one virial radius to the other, and normalized for the projected separation between the two virial radii. We extracted here all pairs of clusters with $M_{100} \geq$ $8 \times 10^{13} M_{\odot}$ and found at a $\leq 4 R_{12}$ distance, both in projection and in 3D (50 pairs in total). We consider here AthenaWFI $1 \mathrm{Ms}$ and $100 \mathrm{ks}$ integrations, XMM $100 \mathrm{ks}$ integration, and eROSITA $20 \mathrm{ks}$ integration, and observations with SKA-LOW, MWA Phase I, and LOFAR-HBA surveys. On average, XMM and eROSITA integrations give a very small detection fraction (we also performed the analysis of a $1.6 \mathrm{ks}$ survey with eROSITA, which yields virtually null detections in the entire sample; see for example the lower right panel of Fig. 16). Athena's $1 \mathrm{Ms}$ integration may instead detect $\sim 10-15 \%$ of the surface of intracluster bridges, but also a $100 \mathrm{ks}$ integration with the same telescope will detect a significant $\sim 5-9 \%$ of it. As expected, the situation is clearly better in the radio domain, as an SKA-LOW survey 


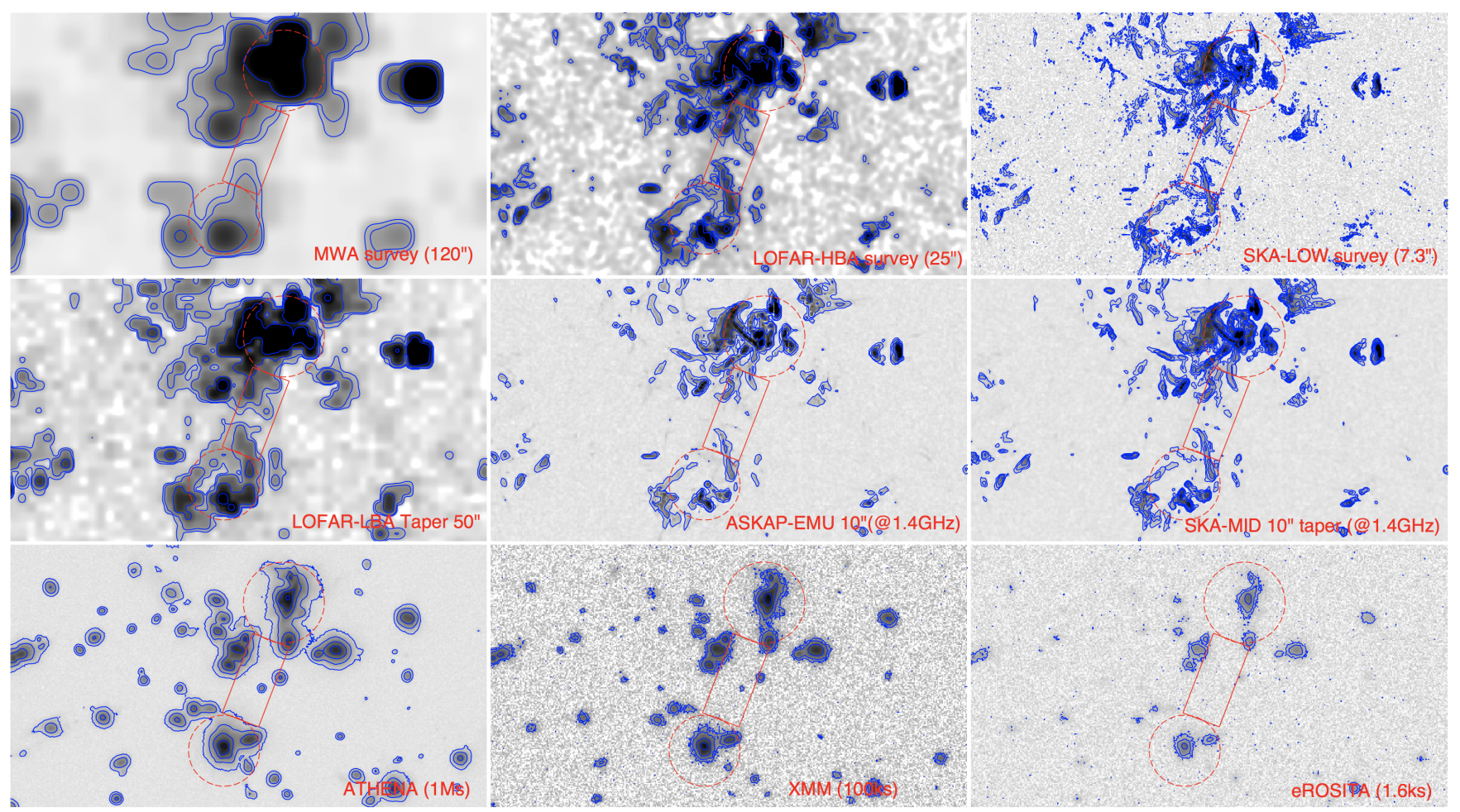

Fig. 16. Example of mock radio (top two rows) and X-ray (bottom row) observations of two bridges connected to a $\sim 2 \times 10^{14} M_{\odot}$ galaxy cluster in our simulation. The red circles denote the $R_{100}$ of the halos connected by bridges with width $5^{\prime}$ (red rectangles), as in Sect. 3.3.3. In all panels, we give in colours the signal and noise of each mock observation, convolved for the resolution of each observation, while the blue contours are drawn with a logarithmic spacing, starting from $3 \mathrm{~S} / \mathrm{N}$ of each observation (see text for explanations).

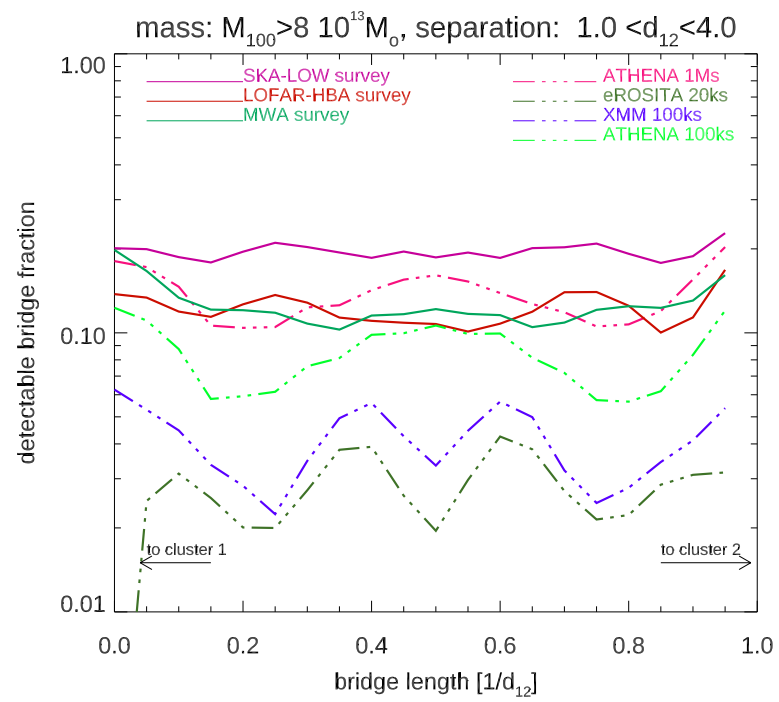

Fig. 17. One-dimensional profile of the detectable bridge fraction connecting pairs of clusters with mass $M_{100} \geq 5 \times 10^{13} M_{\odot}$, considering different X-ray (solid lines) or radio (dot-dashed) observing configurations.

should be able to detect $\sim 20 \%$ of the intracluster filaments area, and MWA Phase I and LOFAR-HBA surveys at least $\sim 10-15 \%$ of it. For radio surveys the detectable (area) fraction is not an unambiguous proxy for the real performance of each telescope, because the rectangular area we consider here is $5^{\prime}$ wide, which is close to the beam size of MWA Phase I observations $\left(2^{\prime}\right)$ or of what is typically achieved for tapered LOFAR-HBA observations $\left(50^{\prime \prime}\right)$. Hence, a few detected beams can contribute to a large covering fraction, yet at the cost of a loss in spatial detail,

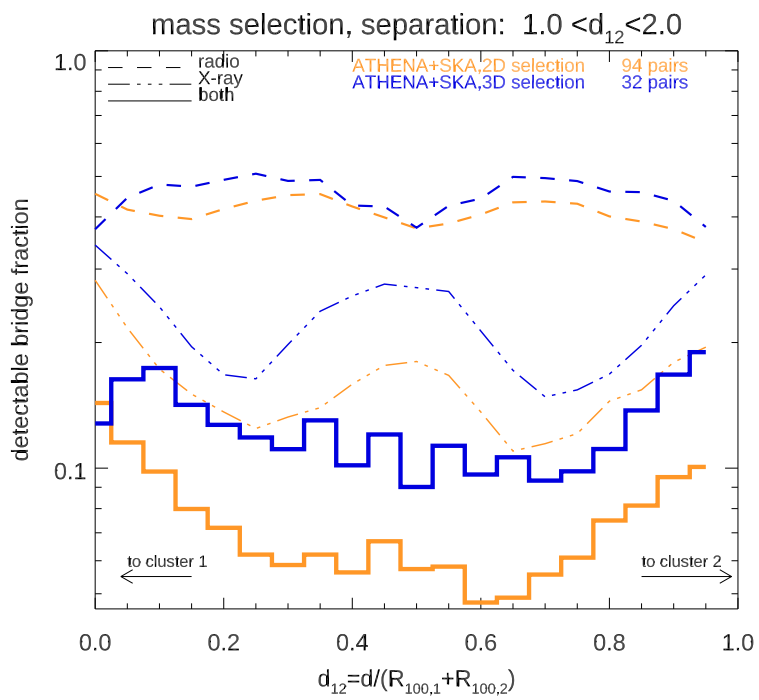

Fig. 18. Detectable fraction of bridges connecting pairs of clusters with mass $M_{100} \geq 10^{13} M_{\odot}$ and for Athena and SKA-LOW observations, for samples of cluster pairs selected only based on their projected (2D) separation, or including also a limit to their physical (3D) separation.

which is crucial (see Fig. 16) if the emission is produced by several shock waves. SKA-LOW will reach the maximum depth after only approximately $10 \mathrm{~h}$ of integration (due to the confusion limit), but it should be able to give detailed spatial information on shocks within the bridge regions, which can also allow a detailed modelling of particle spectra and ageing.

- Separation between cluster pairs: in Fig. 18 we show that if the only prior is on the projected separation (grey lines), a good fraction of the bridge is detectable with a $1 \mathrm{Ms}$ integration with 


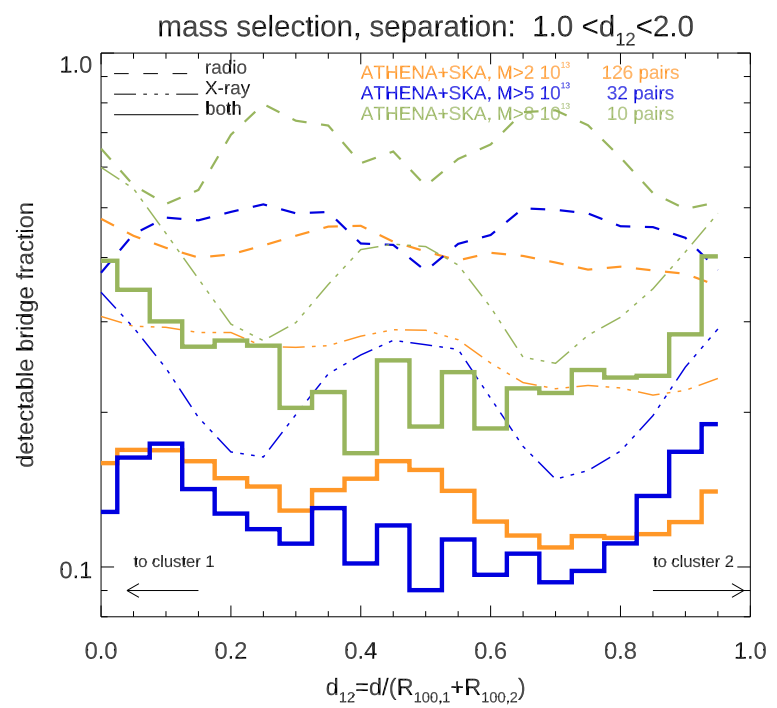

Fig. 19. Detectable fraction of bridges connecting pairs of clusters with mass $M_{100} \geq 10^{13} M_{\odot}$ and for Athena and SKA-LOW observations, for samples of interacting clusters chosen after imposing an increasing lower bound for the minimum mass.

Athena. However, only $\sim 10-20 \%$ is detectable with SKA-LOW, up to a distance of twice $d_{12}$. If we only consider clusters that are physically related, by imposing an additional prior $\left(d_{3 \mathrm{D}} \leq\right.$ $10 d_{12}$ ) on their $3 \mathrm{D}$ distance, the chances of detections in radio, and hence of joint detections, increase very significantly and reach $\sim 15-20 \%$ close to clusters. This follows from the fact that the detectable radio emission is mostly produced by shocks associated with physical gas perturbations triggered during the early merger stage of such objects, rather than to the simple superposition of the shocked outer layers of galaxy clusters that are not interacting;

- Effect of mass selection: in Fig. 19 we show the effect of using a higher mass threshold for the selection of our couples of objects (always connected also in 3D, using the previous prior). The fraction of the joint detection overall increases going to higher masses, even though the total number of objects goes down. For the highest mass selection, $\sim 20 \%$ of the central bridge area (and $\sim 30 \%$ closer to clusters) can be detected, both by Athena and SKA-LOW. We notice that if we repeat the same test assuming a $100 \mathrm{ks}$ survey with XMM-Newton, and a 50" tapered LOFAR-HBA observation, the trend with mass becomes less clear. This is driven by the overall lower rate of detections in $\mathrm{X}$-ray, making this statistics dominated by small number statistics, as shown in Fig. 20. However, the performances of LOFARHBA tapered to this resolution, meant to maximize the signal to noise to diffuse emission, are comparable with an SKA-LOW survey, as they give just a $\sim 25 \%$ smaller fraction of detectable bridge area.

The limited volume of our simulation does not allow us to test a large number of cluster pairs. Hence our work can only identify global trends across the investigated cluster population. We conclude that the best strategy to detect X-ray emission from the WHIM in intracluster bridges is to select cluster pairs with short physical and projected separation $\left(1 \leq d / R_{12} \leq 4\right.$, where $R_{12}=R_{100,1}+R_{100,2}$, with large masses $\left(M_{100} \geq 10^{14} M_{\odot}\right)$ and with the additional prior of having detected radio emission between them. In this case, investing $\sim 1 \mathrm{Ms}$ of observation time with Athena should result in a detection of the WHIM in emission from $\sim 20-30 \%$ of the connecting area, which can in principle be suitable for spectroscopic analysis with X-IFU.

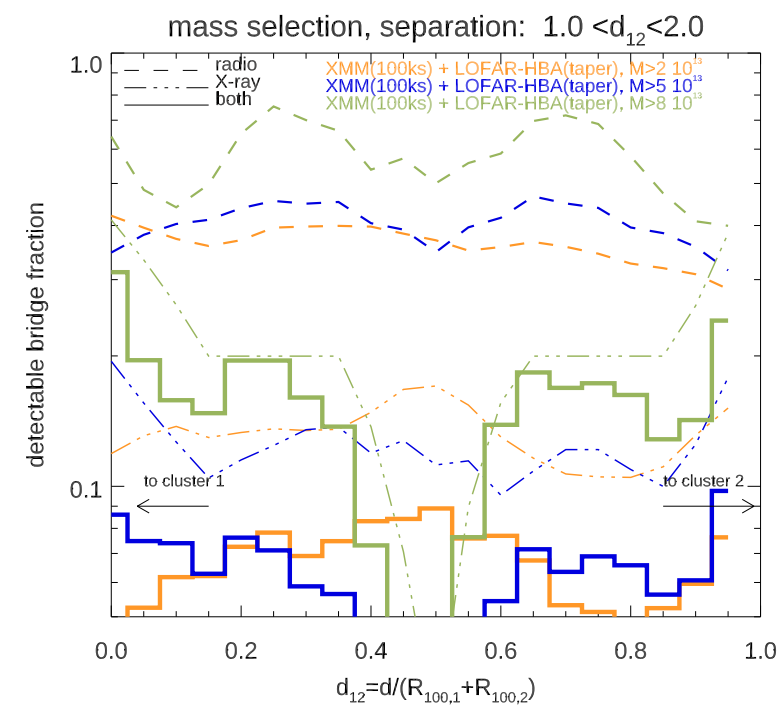

Fig. 20. Detectable fraction of bridges connecting pairs of clusters with different masses, as in Fig. 19, but for $100 \mathrm{ks}$ exposures with XMMNewton and LOFAR-HBA (50" taper) observations.

\subsubsection{Pilot X-IFU observation of a gas bridge between interacting clusters}

Finally, we conducted a pilot study of a potentially promising target for future $1 \mathrm{Ms}$ Athena X-IFU observations, applying the end-to-end X-IFU pipeline outlined in Roncarelli et al. (2018). We performed the ENZO Adaptive Mesh Refinement (AMR) resimulation of a galaxy cluster in an early merger state at $z=0.1$, with a total mass of $M_{100}=6.37 \times 10^{14} M_{\odot}$ and a virial radius of $R_{100}=2.05 \mathrm{Mpc}$.

We selected this cluster from a different parent simulation, using nested initial conditions and seven additional levels of AMR to increase the maximum spatial resolution to $\Delta x_{\max }=$ $3.95 \mathrm{kpc}$, as in Vazza et al. (2018b). This cluster has been specifically selected as at $z=0.1$ it shows an ongoing merger with a second massive galaxy cluster (e.g. Domínguez-Fernández et al. 2019), with an indication of strong dynamical activity in the $R_{100}-R_{200}$ radial range from the first cluster. Moreover, by applying the same mock observation techniques introduced in the previous sections, we identified in this object a patch, located between the two clusters, in which a significant detection will be possible both with SKA-LOW and with a long Athena integration. We also analysed this object using the multi-scale filtering technique to detect shocks and turbulent motions (and further decompose their velocity field into compressive and solenoidal components) as in Vazza et al. (2017b).

As shown in Fig. 21, the region between the two colliding clusters has a temperature of $T \sim 5-6 \mathrm{keV}$, significantly hotter than the surrounding ICM, as an effect of the ongoing merger. Compression rather than shocks is responsible for this heating, as the outer cluster shells of the interacting clusters get compressed at the velocity of $\sim 500-700 \mathrm{~km} \mathrm{~s}^{-1}$ and the average density in the bridge is $\sim 10^{2}\left\langle\rho_{\mathrm{b}}\right\rangle$ (where $\left\langle\rho_{\mathrm{b}}\right\rangle$ is the cosmic mean baryon density). Due to the large temperature and sound speed, no strong accretion shocks are found. Instead, we find a network of $\mathcal{M} \sim 2-5$ shocks, intermittently driven by the event. Our turbulent filtering detects turbulent velocities on the order of $\sim 400-500 \mathrm{~km} \mathrm{~s}^{-1}$ in this region, almost equally divided into solenoidal and compressive components, with a typical outer scale of $\sim 400 \mathrm{kpc}$. The magnetic field in the bridge region where radio and X-ray emission can be detected is $B \sim 0.1-0.2 \mu \mathrm{G}$, 

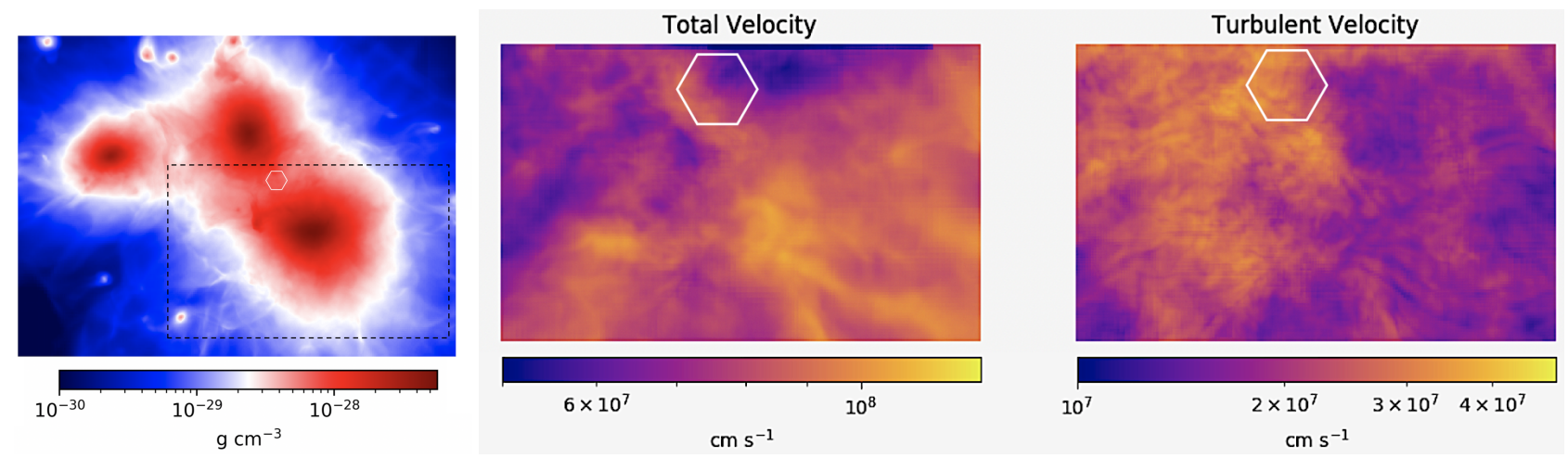

Fig. 21. Left panel: projected mean gas density for the interacting cluster used for our SIXTE simulation in Sect. 3.3.4. Central and right panels: projected (volume weighted) map of total gas velocity module and of turbulent velocity module for a zoomed region in the left panel. The additional white region shows the $5^{\prime} \times 5^{\prime}$ region used for our SIXTE mock observation.
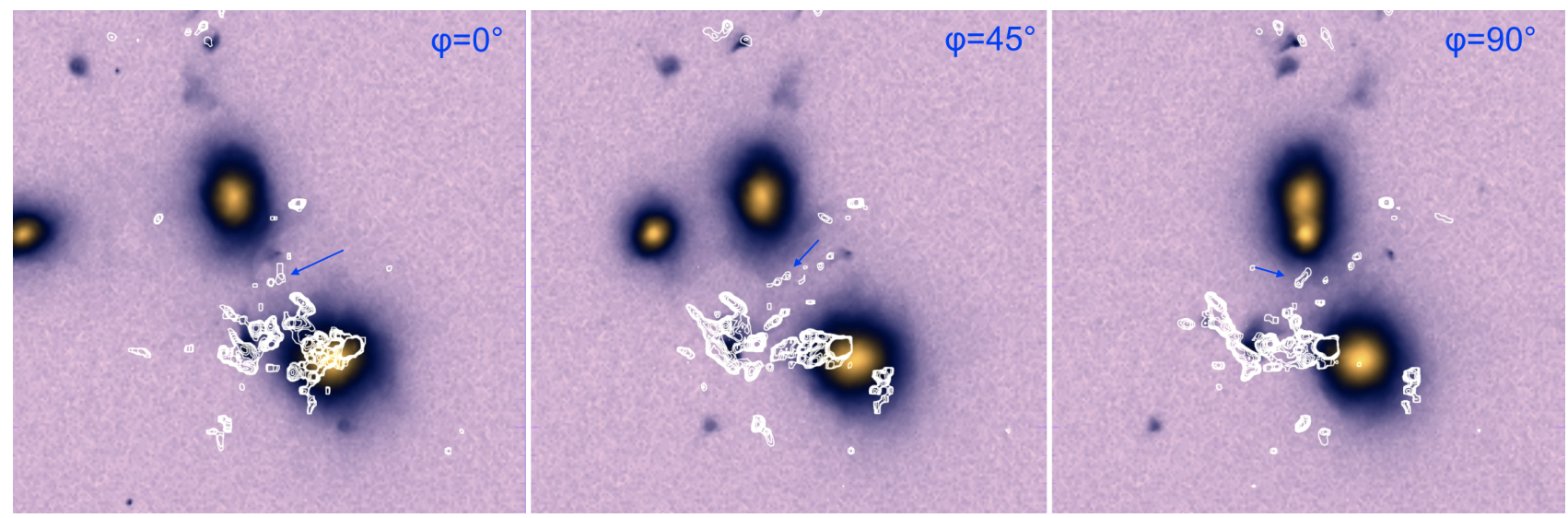

Fig. 22. Three different rotations $\left(0^{\circ}, 45^{\circ}\right.$, and $\left.90^{\circ}\right)$ around cluster E5A, showing the X-ray counts (colours) and the detectable radio emission at $260 \mathrm{MHz}$ (contours). The additional blue arrow gives the approximate location of the detectable emission region in the rotated images.

which yields a plasma beta parameter of $\beta_{\mathrm{pl}}=n k_{\mathrm{b}} T /\left(B^{2} / 8 \pi\right) \sim$ 5000 .

From this region, we extracted a $(75 \times 75 \times 4800) \mathrm{kpc}^{3}$ box that contains the entire emitting regions, as well as the outer regions of the two clusters in the field of view. The region's $z$-axis is aligned with the line of sight. Following Roncarelli et al. (2018), we simulated with $1 \mathrm{eV}$ resolution the X-ray emission assuming an APEC model (version 2.0.2, Smith et al. 2001), absorbed with a Galactic equivalent hydrogen column density of $n_{\mathrm{H}}=2 \times 10^{20}$ atoms cm $\mathrm{cm}^{-2}$. We assume a rather conservative value of (spatially constant) metal abundance $Z=0.2 Z_{\odot}$ with respect to the solar abundance in Anders \& Grevesse (1989). To provide a realistic observational setup, we included an X-ray background modelled by (i) two thermal local components with temperatures of 0.099 and $0.225 \mathrm{keV}$ and normalization for the adopted APEC model of $1.535 \times 10^{-6}$ per $\operatorname{arcmin}^{2}$ of integrated area, respectively, both with solar metallicity. Only the second component is absorbed by $n_{\mathrm{H}}$ and has a normalization reduced by a factor of 0.42 (see McCammon et al. 2002). (ii) A cosmic X-ray background, assumed to be unresolved at the $20 \%$ level, simulated with an absorbed power law with a photon index of 1.5 and a normalization of $2.1 \times 10^{-7}$ photons $\mathrm{keV}^{-1} \mathrm{~cm}^{-2} \mathrm{~s}^{-1} \mathrm{arcmin}^{-2}$ at $1 \mathrm{keV}$ (see also e.g. Lotti et al. 2014). After projecting these spectra into the plane of the sky, we used the resulting signal as an input for the SIXTE simulator ${ }^{9}$ (Schmid et al. 2013),

\footnotetext{
9 https://www.sternwarte.uni-erlangen.de/research/ sixte/
}

assuming $1 \mathrm{Ms}$ exposure. An updated instrumental particle background (Lotti et al. 2017) is already implemented in SIXTE. The output of the SIXTE code consists of a realistic mock X-IFU observation, that is, an observed event-list containing a total of $\sim 16500$ counts $^{10}(0.3-10 \mathrm{keV})$ from a $0.382 \operatorname{arcmin}^{2}$ area in the centre of the X-IFU field. These photons were folded in a single spectrum that has been jointly fitted with a spectrum containing only the expected photons from a background modelled as described above, using a Cash statistic (Cash 1979) implemented in XSPEC (Arnaud 1996). To derive the thermodynamic and kinematic properties of the gaseous component associated to the cluster, we assumed a BAPEC model with five free parameters, namely normalization, temperature, metal abundance, redshift, and velocity dispersion ( $n_{\mathrm{H}}$ has been fixed to the input value) and proceeded with a blind fitting analysis.

In Fig. 23, we show the mock spectra and the fitting results, with the three components (thermal, X-ray, and particle background). Togethe with the reference values, the results of the X-IFU spectral fitting simulation are also shown in Table 3, computed as the averages on the simulation cells. Thanks to the $1 \mathrm{Ms}$ exposure, temperature (compared to the spectroscopic-like) and metallicity are recovered with high precision with a typical

\footnotetext{
${ }^{10}$ As a further reference to the reader, we verified that $\sim 8900$ counts are coming from the source (i.e. the simulated plasma), while $\sim 5400$ and 2200 come from the X-ray and particle background, respectively. This piece of information, which would not be available to the observer, has been omitted in the following analysis.
} 

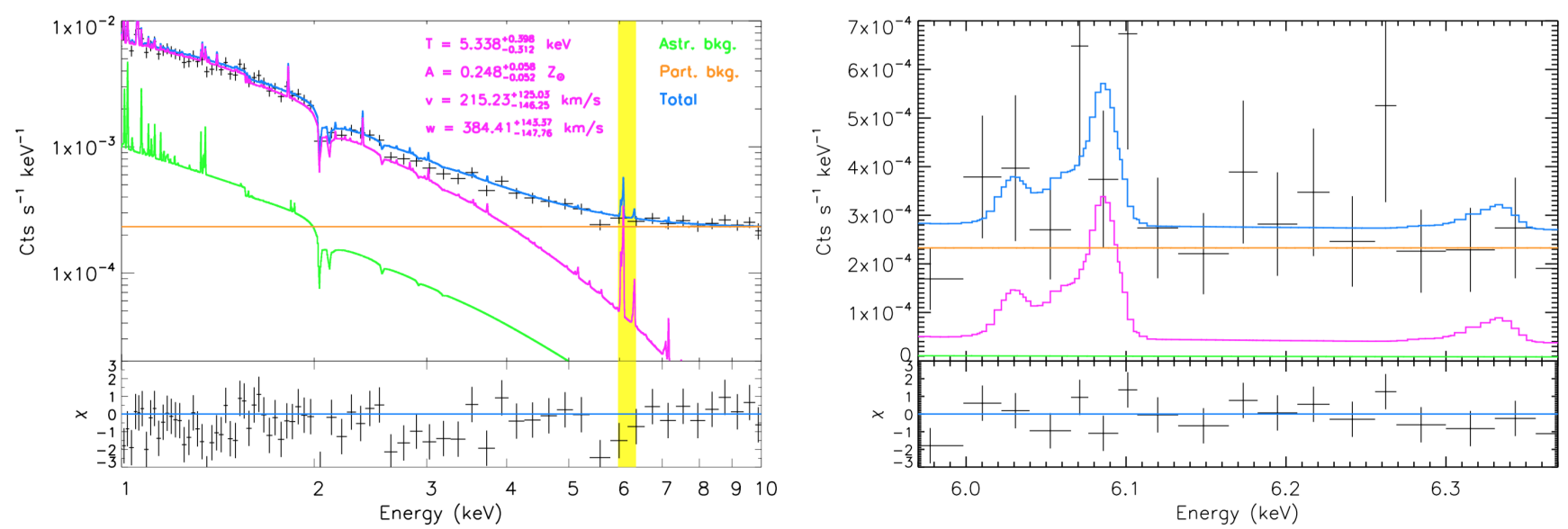

Fig. 23. SIXTE simulations of one simulated bridge in between galaxy clusters. Left panel: X-IFU simulated spectrum of the gas bridge and its spectral analysis. Spectral data points with error bars (black crosses) are shown with the best-fit model (blue solid line) and its three components: thermal emission from the gas (magenta), X-ray astrophysical background (green), and particle background (orange). Fit results, with errors, for the four physical quantities of the gas components are also shown. Bottom sub-panel: residuals with respect to the model. Right panel: same as left panel, but zooming-in on the 6-6.4 keV energy range (highlighted in yellow in the left panel) where the most prominent emission lines (blended Fe xxv and Fe xxvi K complexes) are present. In both panels data points have been rebinned for display purposes. The plot scaling for the spectra is logarithmic and linear in the left and right panels, respectively.

Table 3. Physical properties of the plasma computed in the simulation volume compared to the ones measured through the end-to-end X-IFU simulation for a $1 \mathrm{Ms}$ integration with spectral fitting.

\begin{tabular}{lcc}
\hline \hline Field & Input (sim) & Output (X-IFU) \\
\hline$T(\mathrm{keV})$ & 5.52 & $5.33 \pm[-0.31,0.40]$ \\
$v$ & 65 & $215 \pm[-146,123]$ \\
$w$ & 465 & $384 \pm[-148,144]$ \\
$A\left(1 / Z_{\odot}\right)$ & 0.20 & $0.25 \pm[-0.05,0.06]$ \\
\hline
\end{tabular}

Notes. The reference (input) temperature is spectroscopic-like, $v$ and $w$ are the emission weighted averages, and $A$ is the metal abundance, computed in the simulation volume as in Roncarelli et al. (2018).

statistical error of $0.3-0.4 \mathrm{keV}$ and $0.05 Z_{\odot}$, respectively. Most importantly, the evidence of a significant velocity dispersion is detected at high significance (more than $2.5 \sigma$ ), albeit with a relatively high statistical error. All the quantities are recovered with no apparent systematic bias.

However, our setup assumes a perfectly known (X-ray and particle) background. To relax this assumption, we also ran 100 Monte-Carlo spectral simulations that assumed the cluster component and propagated random fluctuations that are consistent with the current expectations on the background reproducibility in X-ray. We ran these simulations on $n_{\mathrm{H}}$ (at $1 \%$ level), on the particle background (2\%), and on the remaining parameters of the background model, which are the thermal components and the power law for the unresolved cosmic X-ray background $(5 \%)$. We estimate the following systematic scatter in the distribution on the best-fitting measurements of the five parameters of the BAPEC model: $\sim 2 \%, 6 \%, 27 \%, 58 \%$, and $<1 \%$ on normalization, temperature, metal abundance, line broadening, and redshift, respectively. These results show that, while most of the parameters will be only limited by the photon counts statistic, the characterization of the emission lines in terms of total metallicity and broadening at this level of surface brightness will depend on a reliable modelling of the underlying background.

These first results offer an interesting physical application to the study of shock waves and particle acceleration in the periphery of galaxy clusters: namely a new method to constrain the shock Mach number based on spectroscopic analysis. If we assume that the measured velocity dispersion is similar to the velocity jump induced by the shock, $w \approx \Delta v^{\prime}=\Delta v / \cos \phi$ (in which $\phi$ is the inclination of the shock normal with respect to the line of sight), and that the local sound speed is given by the temperature probed by the spectroscopy, through the "velocityjump" method (Vazza et al. 2009) we derive

$\mathcal{M}_{\mathrm{XIFU}}=\frac{2}{3}\left(\frac{\Delta v^{\prime}}{c_{\mathrm{s}}}+\sqrt{\frac{4 \Delta v^{\prime}}{c_{\mathrm{s}}}+9}\right)$,

which yields $\mathcal{M}_{\mathrm{XIFU}} \approx 2.42$ for the X-IFU best fit values of $T \approx 5.33 \mathrm{keV}, w \approx 465 \mathrm{~km} \mathrm{~s}^{-1}$, and considering $\phi \approx 75^{\circ}$ for the shock normal (as suggested by Fig. 22, right panel). This is not too different from the $\mathcal{M} \sim 2.5-3$ range of Mach number we can measure in 3D within the X-IFU's field of view, and suggests the interesting possibility of an independent way of constraining the shock parameters via spectroscopic analysis, which can be combined and compared with the available radio information there (e.g. Mach number estimated from radio spectral indices for high resolution observations and particle acceleration efficiency). We comment that intracluster bridges, given their particular geometrical selection, may allow the performance of this study in a more robust way than in more internal regions of galaxy clusters where radio relics are found. This is because in these external regions the local sound speed is low enough and the velocity field is large enough that the Doppler broadening due to shocks with a large inclination along the line of sight may be clearly detected via spectroscopic analysis.

\section{Discussion: physical and numerical uncertainties}

Our results are based on non-radiative MHD simulations, in which the role of galaxy formation processes is neglected. This introduces a number of caveats due to the lack of energy losses (e.g. radiative cooling processes) and feedback processes.

In general, we expect the most relevant effects of cooling and feedback to be limited to cluster centres. Meanwhile 

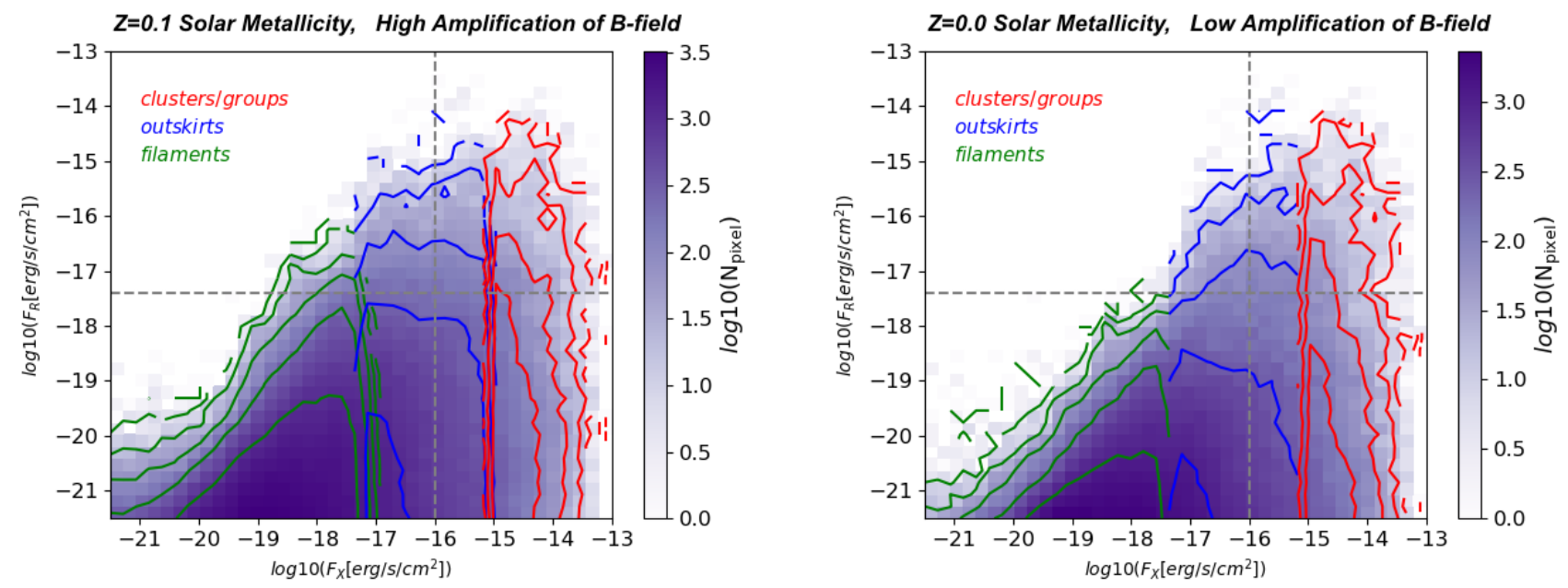

Fig. 24. Distribution of X-ray and radio flux for variations of our sky model. Left panel: we considered a "high amplification model" for the magnetic field and we assumed a uniform gas metallicity of $Z=0.1 Z_{\odot}$ everywhere (instead of $Z=0.3 Z_{\odot}$ as in our baseline model); in the right panel we considered a low-amplification model and a zero metallicity everywhere. The meaning of colours and contours is as in Fig. 5. The text gives more details on the model variations.

for $\leq 0.2 R_{100}$ the combined effect of cooling, star formation, and feedback (stellar or AGN ones) can introduce a significant amount of clumping in clusters (Roncarelli et al. 2006b; Nagai \& Lau 2011), as well as increase the gas density in cluster cores by approximately ten times compared to non-radiative simulations. However, such effects are predicted to be negligible at distances larger than $\sim R_{100}$ (e.g. Vazza et al. 2013), as well as within intracluster bridges.

The absence of galaxy formation processes limits our ability to model the $3 \mathrm{D}$ distribution of metals in the outer regions of galaxy clusters. Hence, we assume a uniform $Z=0.3 Z_{\odot}$ gas metallicity. The assumption of uniform metallicity for all elements reduces the number of free parameters making the spectral fitting presented in Sect. 3.3.4 easier. In reality, as pointed out by Cucchetti et al. (2018), the X-IFU spectral analysis will require a detailed treatment of the various emitting ions due to the chemical complexity of the ICM (see also Roncarelli et al. 2010; Biffi et al. 2013). However, most of the information on $w$ is encoded in the Fe Xxv and Fe XxvI $\mathrm{K}$ complexes lines (see the right panel of Fig. 23), so this simplification has a minor impact on the accuracy of our results.

In Fig. 24 we present a few variations of our model, concerning the assumed gas metallicity or the magnetic field strength in our simulation. A comparison of the two panels of Fig. 24 with Fig. 5 shows that the impact of metal line emission on the detectability of cluster outskirts is small, as the blue contours barely change. The reason for this is that in the $\sim 10^{7} \mathrm{~K}$ temperature range of intracluster bridges the line emission only accounts for a few percent of the total X-ray emission in the $0.8-1.2 \mathrm{keV}$ band of Athena-WFI.

More critical is the level of gas metallicty for any spectrosopic attempt at characterizing the local plasma condition, as in our mock X-IFU observation described in Sect. 3.3.4. However, in this case we already used the more conservative value of $0.2 Z_{\odot}$ (spatially uniform everywhere) for our $1 \mathrm{Ms}$ mock XIFU observation. Also in this case, a robust measurement of local plasma parameters is possible, provided that the particle and instrumental background are understood. Our tests also indicate that conversely if $Z \geq 0.1 Z_{\odot}$ in intracluster bridges, the reconstruction process of gas conditions through spectroscopic analysis (even with a $1 \mathrm{Ms}$ integration) will be unreliable and dominated by large uncertainties. However, a $\sim 0.1 Z_{\odot}$ metallicity is probably even too conservative, and larger metallicity values are likely present in this environment (e.g. Biffi et al. 2018; Mernier et al. 2018).

Recently, Khabibullin \& Churazov (2019) reconsidered the contribution from the resonantly scattered cosmic X-ray background to the line emission for the WHIM. Resonant scattering can increase the emissivity of the WHIM considered in this work by a factor of $\sim 30$. However, this boost is limited to the gas at $T \leq 10^{6} \mathrm{~K}$ and, when integrated in the [0.5-1] keV band, it is on the order of $\sim 4$ for the coldest part of the WHIM only. Therefore, this effect is not expected to contribute to the detectability of the much hotter gas located in intracluster bridges.

Next, we tested realistic variations of the magnetic field model, which affects the level of predicted synchrotron emission (see Fig. 24). Magnetic fields may in principle be overestimated in cosmic filaments, in case the seed fields are not of primordial origin and/or there is no dynamo amplification capable of increaseing the magnetization of the WHIM to the $\sim 10 \mathrm{nG}$ level (Vazza et al. 2017a). Conversely, limited to the environment of intracluster bridges, our AMR simulation can resolve the ongoing dynamo amplification of seed fields, albeit with final field strengths that are typically far from equipartition with the kinetic energy, at least in our simulation (e.g. Locatelli et al. 2018). The contribution from un-resolved gas motions by the finite numerical resolution in our scheme may underestimate the level of small-scale dynamo amplification, which becomes independent from the amplitude of seed fields for large enough Reynolds number (e.g. Cho 2014; Beresnyak \& Miniati 2016). If this is the case, the $\sim 0.1-0.2 \mu \mathrm{G}$ we measured for our intracluster bridge in Sect. 3.3.4 may be underestimated, even if to our knowledge this run is the most resolved so far for objects of this kind.

To bracket uncertainties, we followed Vazza et al. (2015b) and used a post-processing method to model two extreme scenarios for the amplification of magnetic fields in the cosmic web: a high-amplification model (HA) and a low-amplification model (LA). In both scenarios, the magnetic field strength from the simulation is renormalized a-posteriori, depending on the local gas overdensity. In the HA model, we account for the efficient magnetization of all cosmic gas denser than the critical gas mean density $(\rho \geq\langle\rho\rangle)$, which cannot be resolved in the simulation. 
Conversely, in the LA case we assume that the amplification can be efficient only for densities larger than that of virialized halos $(\rho \geq 300\langle\rho\rangle)$. In both cases, the post-processing normalization is such that the magnetic field energy within each cell becomes equal to $1 \%$ of the cell thermal energy, if the gas density in the cell falls within the HA or LA overdensity range, or remains the original one from our baseline MHD simulation. As discussed in Vazza et al. (2015b), the two models produce very different radio fluxes, while they yield similar fluxes in the inner parts of clusters. The two panels in Fig. 24 show that the radio flux from filaments is $\geq 10$ times larger if magnetic fields are much more amplified in filaments than our simulation can resolve. On the other hand, the predicted radio emission from cluster outskirts and from the innermost cluster regions would be only mildly increased (factors $\leq 4-5$ on average) because, for most radio bright shocks, the magnetic energy in our simulation is not far from being $\sim 1 \%$ of the thermal gas energy there.

For the electron acceleration, our model only includes direct acceleration of electrons from the thermal pool (Hoeft \& Brüggen 2007), but it neglects the effect of shock re-accelerated electrons (Pinzke et al. 2013; Kang et al. 2012). However, the density of fossil electrons in cluster outskirts and in bridges is largely uncertain and any level of fossil electrons will increase the emission beyond our estimates. Also, in our model we neglected for simplicity any further injection or re-acceleration of electrons by additional processes (e.g. turbulent reacceleration, re-connection, shock drift acceleration; see e.g. Brunetti \& Jones 2014 and Bykov et al. 2019 for recent reviews), which, especially at low frequency, may power the emission beyond our estimates. Recent LOFAR-HBA observations suggest indeed that more volume filling and diffuse radio emission processes may be present on scales of Megaparsecs out outside of galaxy clusters. Given the above issues, the X-ray and radio emission model considered in the main paper represents a conservative lower limit on the joint detectability of intracluster bridges and extreme cluster outskirts.

\section{Conclusions}

We have presented mock radio and X-ray observations of the cosmic web, based on recent cosmological simulations obtained with the cosmological MHD code ENZO (Bryan et al. 2014). Extending our first exploratory study in Cassano et al. (2018), we quantified the chances of "double detecting" the shocked cosmic web in both frequency ranges, and propose best observing strategies tailored for future instruments (e.g. SKA and Athena). Our study highlights that the most promising targets for double detections outside of galaxy clusters are typically located in matter "bridges" connecting pairs of galaxy clusters in an earlier merger state. At this interaction stage, both radio and $\mathrm{X}$-ray emission are boosted compared to the more typical conditions found in cluster outskirts. Such (transient) excess emissions appear to be within the detection range of existing (LOFAR, MWA, ASKAP) and future (SKA-LOW and SKA-MID) radio surveys, as well as of very long $(\geq 100 \mathrm{ks})$ integrations with Athena, XMM, and eROSITA. Based on our simulations, the chances of double detections get greatly increased for pairs of objects with a physical (3D) association, with masses in excess of $\geq 10^{14} M_{\odot}$ and with a projected separation between one and four mean virial diameters (e.g. the sum of the two virial radii of interacting clusters). For practical purposes, a prior detection of such bridges in the radio domain is expected to serve as a strong indication of the possibility of detecting emission also in the soft $(\leq 1.2-2 \mathrm{keV}) \mathrm{X}$-ray band.
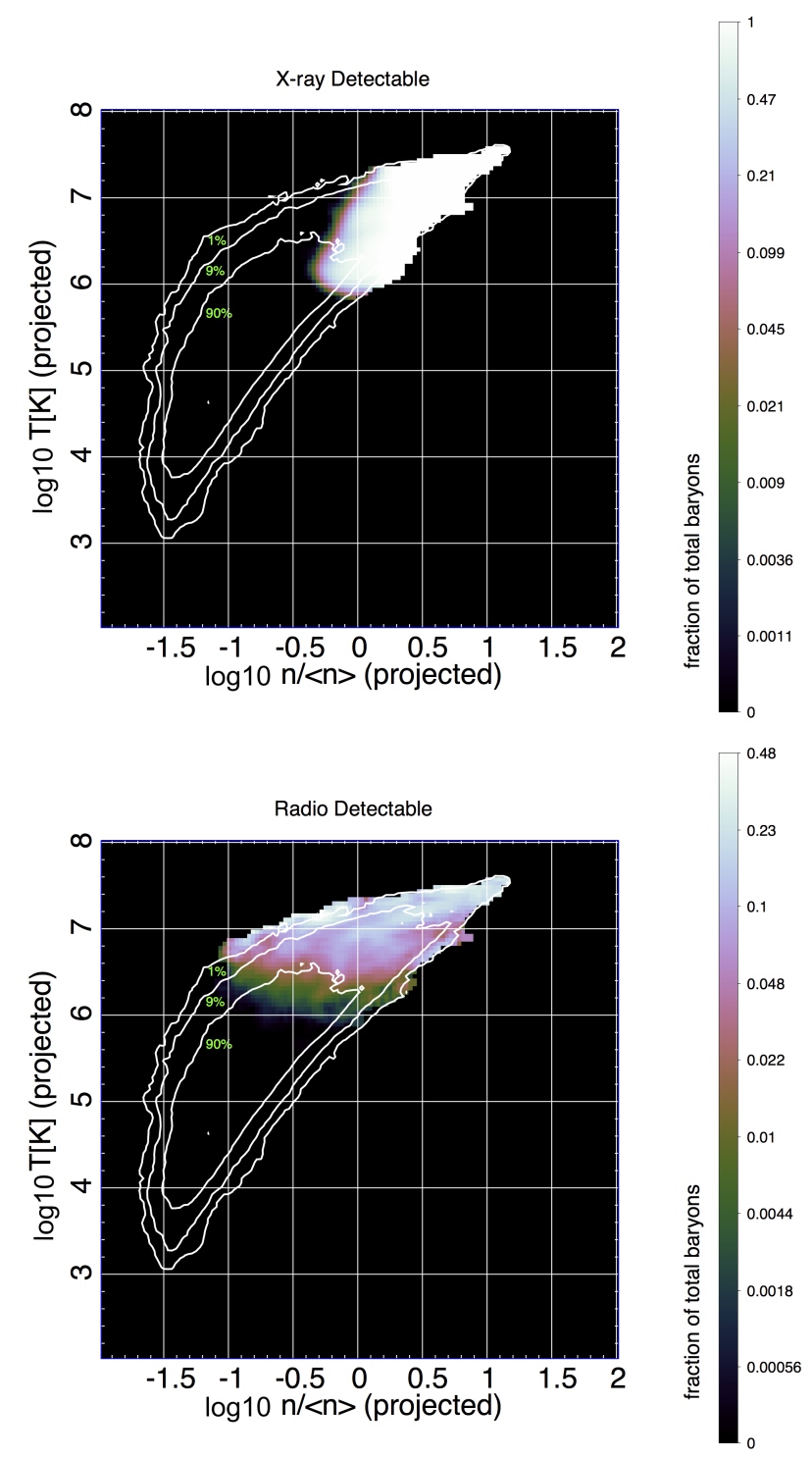

Fig. 25. Distribution of projected mean temperature and density for all pixels in our sky model at $z=0.05$. The total area within the isocontours marks the area where the $90 \%, 9 \%$, and $1 \%$ of the baryon budget is contained. The colours mark the fraction of the total baryon budget that can be detected with X-ray detections with Athena (top panel) or with radio detections with SKA-LOW (bottom panel).

Detecting radio emission from transient shocks in such systems will also represent a strong motivation to attempt long dedicated integrations with Athena's X-IFU. For example, a deep ( $\sim 1 \mathrm{Ms})$ integration with X-IFU on such a jointly detectable portion of intracluster bridges should represent a new strong scientific case to study plasma shock physics in the rarefied environment of the WHIM. This is made possible by enabling the derivation of the shock Mach number entirely from spectroscopically-derived information of the local gas velocity dispersion and of the local sound speed, in a temperature regime that is difficult to find in galaxy clusters (Sect. 3.3.4).

Closely interacting pairs of galaxy clusters have already been detected, and observations have highlighted unexpected thermal and non-thermal gas features in the interaction region of galaxy clusters at an early merging state (e.g. Akamatsu et al. 2017; Sugawara et al. 2017; Caglar \& Hudaverdi 2017; Botteon et al. 2018; Bonafede et al. 2018; Alvarez et al. 2018). 
Also Sunayaev-Zeldovich observations of close pairs have hinted at the presence of dense and X-ray undetectable gas in such associations (Planck Collaboration VIII 2013; Bonjean et al. 2018).

Such objects are clearly different from cosmic filaments that are produced by simulations on much larger scales (e.g. Gheller et al. 2016). Moreover, the gas in these bridges is on a thermodynamical state that is different from the standard WHIM, as the typical density and temperature values are a factor of $\sim 10$ larger than in the WHIM, and are more in the ICM regime. However, simulations suggest that bridges are relatively short lived $(\leq \mathrm{Gyr})$, and should undergo a fast evolution compared to filaments on a larger scale. They also suggest that bridges used to be part of the standard WHIM 1 Gyr ago, before becoming observable in the $\mathrm{X}$-ray band. For this reason they have the potential of illuminating an important intermediate stage in the evolution of cosmic baryons, where gas that has been only shock-heated once in the past becomes more quickly advected onto larger clusters and is subject to large-scale mixing, reprocessing by weaker shocks, and supersonic turbulence. This leads to a transient, "boosted" WHIM phase, with a mean temperature beyond the canonical (but not entirely physically motivated) temperature bounds associated to the WHIM $\left(10^{5} \mathrm{~K} \leq T \leq 10^{7} \mathrm{~K}\right)$.

In summary, our work stresses the importance of the radio band in studying the missing baryons of the cosmic web. We quantify this by presenting in Fig. 25 the distribution of the mean temperature and gas over density for all pixels in our sky model ${ }^{11}$. Contours denote the total fraction of baryons at $z=0.05$. Overlaid is the fraction of baryons that should be detectable by X-ray observations using a $1 \mathrm{Ms}$ exposure with Athena, or with the SKA-LOW survey at $260 \mathrm{MHz}$.

Clearly, X-ray observations are most efficient in the hightemperature and high-density part of the plot. However, less than $10 \%$ of the total budget of baryons in the Universe are located in this range. Conversely, radio surveys can trace only a much a smaller fraction of hot and dense baryons in the Universe, due to the small filling factor of shocks leading to radio emission in this regime. However, radio detections trace baryons with significantly lower projected temperature and density compared to $\mathrm{X}$-ray observations, enabling them to probe into the gas phase where $\sim 90 \%$ of cosmic baryons are located. As such detections can only illuminate the shocked portion of the WHIM (or immediately downstream of it), it will be crucial to assess the bias factor between the radio emitting fraction of the cosmic web and its larger ("radio quiet") component. With the assistance of advanced numerical simulations capable of assessing this bias as a function of environment, wide and deep radio surveys will have the potential to convert systematic detections of radio shocks in the rarefied cosmic web into an estimate of the amount of missing baryons in the Universe.

Acknowledgements. We thank our anonymous referee for the fruitful feedback on the first version of the paper, which has led to an improved presentation of our results. The cosmological simulations were performed with the ENZO code (http://enzo-project.org), which is the product of a collaborative effort of scientists at many universities and national laboratories. We gratefully acknowledge the ENZO development group for providing extremely helpful and well-maintained online documentation and tutorials. F.V. acknowledges financia support from the ERC Starting Grant "MAGCOW", no. 714196. We acknowledge the usage of computational resources on the Piz Daint supercomputer at CSCS-ETHZ (Lugano, Switzerland) under projects s701 and s805 and at the Jülich Supercomputing Centre (JFZ) under project HHH42. We also acknowl-

11 As noted in Sect. 3.1, the mass-weighted temperature and volume-weighted gas density here underestimate the corresponding three-dimensional values (where most of the emission along the LOS is produced) by a factor of $\sim 10$. edge the usage of online storage tools kindly provided by the Inaf Astronomica Archive (IA2) initiave (http://www.ia2 .inaf.it). S. E. and M. R. acknowledge funding from the European Union's Horizon 2020 Programme under the AHEAD project (grant agreement n. 654215). S.E. acknowledges financial contribution from the contracts NARO15 ASI-INAF I/037/12/0, ASI 2015-046-R.0, and ASI-INAF n.2017-14-H.0. We acknowledge fruitful scientific feedback by A. Bonafede, M. Cappi, M. Markevitch, N. Locatelli, R. Cassano, G. Brunetti, I. Prandoni, E. Churazov, and I. Khabibullin, which improved the presentation of our results. We also thank T. Boller and V. Ghirardini for having provided useful information on the performance of eROSITA and XMM-Newton, respectively.

\section{References}

Akamatsu, H., Fujita, Y., Akahori, T., et al. 2017, A\&A, 606, A Alvarez, G. E., Randall, S. W., Bourdin, H., Jones, C., \& Holley-Bockelmann, K. 2018, ApJ, 858, 44

Anders, E., \& Grevesse, N. 1989, Geochim. Cosmochim. Acta, 53, 197

Arnaud, K. A. 1996, in Astronomical Data Analysis Software and Systems V, eds. G. H. Jacoby, \& J. Barnes, ASP Conf. Ser., 101, 17

Bagchi, J., Enßlin, T. A., Miniati, F., et al. 2002, New Astron., 7, 249

Barcons, X., Barret, D., Decourchelle, A., et al. 2012, ArXiv e-prints [arXiv:1207.2745]

Bartels, R., Zandanel, F., \& Ando, S. 2015, A\&A, 582, A20

Beresnyak, A., \& Miniati, F. 2016, ApJ, 817, 127

Biffi, V., Dolag, K., \& Böhringer, H. 2013, MNRAS, 428, 1395

Biffi, V., Mernier, F., \& Medvedev, P. 2018, Space Sci. Rev., 214, 123

Bonafede, A., Vazza, F., Brüggen, M., et al. 2013, MNRAS, 433, 3208

Bonafede, A., Brüggen, M., Rafferty, D., et al. 2018, MNRAS, 478, 2927

Bonaldi, A., \& Brown, M. L. 2015, MNRAS, 447, 1973

Bonjean, V., Aghanim, N., Salomé, P., Douspis, M., \& Beelen, A. 2018, A\&A, 609, A49

Borm, K., Reiprich, T. H., Mohammed, I., \& Lovisari, L. 2014, A\&A, 567, A65 Botteon, A., Shimwell, T. W., Bonafede, A., et al. 2018, MNRAS, 478, 885

Brown, S. D. 2011, A\&A, 32, 577

Brunetti, G., \& Jones, T. W. 2014, Int. J. Mod. Phys. D, 23, 1430007

Bryan, G. L., Norman, M. L., O'Shea, B. W., et al. 2014, ApJS, 211, 19

Bykov, A. M., Vazza, F., Kropotina, J. A., Levenfish, K. P., \& Paerels, F. B. S. 2019, Space Sci. Rev., 215, 14

Caglar, T., \& Hudaverdi, M. 2017, MNRAS, 472, 2633

Cappelluti, N., Ranalli, P., Roncarelli, M., et al. 2012, MNRAS, 427, 651

Cash, W. 1979, ApJ, 228, 939

Cassano, R., Ettori, S., Giacintucci, S., et al. 2010, ApJ, 721, L82

Cassano, R., Fender, R., Ferrari, C., et al. 2018, ArXiv e-prints [arXiv: 1807.09080 ]

Cen, R., \& Chisari, N. E. 2011, ApJ, 731, 11

Cen, R., \& Ostriker, J. P. 1999, ApJ, 514, 1

Cho, J. 2014, ApJ, 797, 133

Corasaniti, P. S., Ettori, S., Rasera, Y., et al. 2018, ApJ, 862, 40

Cucchetti, E., Pointecouteau, E., Peille, P., et al. 2018, A\&A, 620, A173

Davé, R., Cen, R., Ostriker, J. P., et al. 2001, ApJ, 552, 473

de Graaff, A., Cai, Y.-C., Heymans, C., \& Peacock, J. A. 2019, A\&A, 624, A48

Dedner, A., Kemm, F., Kröner, D., et al. 2002, J. Comput. Phys., 175, 645

Domínguez-Fernández, P., Vazza, F., Brüggen, M., \& Brunetti, G. 2019, MNRAS, 486, 623

Eckert, D., Molendi, S., Vazza, F., Ettori, S., \& Paltani, S. 2013, A\&A, 551, A22

Eckert, D., Jauzac, M., Shan, H., et al. 2015, Nature, 528, 105

Eckert, D., Ghirardini, V., Ettori, S., et al. 2019, A\&A, 621, A40

Ettori, S., Ghirardini, V., Eckert, D., et al. 2019, A\&A, 621, A39

Farnsworth, D., Rudnick, L., Brown, S., \& Brunetti, G. 2013, ApJ, 779, 189

Gheller, C., Vazza, F., Brüggen, M., et al. 2016, MNRAS, 462, 448

Ghirardini, V., Eckert, D., Ettori, S., et al. 2019, A\&A, 621, A41

Giovannini, G., Bonafede, A., Feretti, L., Govoni, F., \& Murgia, M. 2010, A\&A, 511, L5

Govoni, F., Orrù, E., Bonafede, A., et al. 2019, Science, 364, 981

Hickox, R. C., \& Markevitch, M. 2007, ApJ, 671, 1523

Hoeft, M., \& Brüggen, M. 2007, MNRAS, 375, 77

Iapichino, L., Schmidt, W., Niemeyer, J. C., \& Merklein, J. 2011, MNRAS, 414, 2297

Kang, H., Ryu, D., \& Jones, T. W. 2012, ApJ, 756, 97

Keshet, U., Waxman, E., \& Loeb, A. 2004, ApJ, 617, 281

Khabibullin, I., \& Churazov, E. 2019, MNRAS, 482, 4972

Kolodzig, A., Gilfanov, M., Hütsi, G., \& Sunyaev, R. 2018, MNRAS, 473, 4653

Locatelli, N., Vazza, F., \& Domínguez-Fernández, P. 2018, Galaxies, 6, 128

Loi, F., Murgia, M., Govoni, F., et al. 2019, MNRAS, 485, 5285

Lotti, S., Cea, D., Macculi, C., et al. 2014, A\&A, 569, A54

Lotti, S., Mineo, T., Jacquey, C., et al. 2017, Exp. Astron., 44, 371 
Martizzi, D., Vogelsberger, M., Artale, M. C., et al. 2019, MNRAS, 486, 3766 McCammon, D., Almy, R., Apodaca, E., et al. 2002, ApJ, 576, 188

Mernier, F., Biffi, V., Yamaguchi, H., et al. 2018, Space Sci. Rev., 214, 129

Mushotzky, R. F., Aird, J., Barger, A. J., et al. 2019, ArXiv e-prints [arXiv:1903.04083]

Nagai, D., \& Lau, E. T. 2011, ApJ, 731, L10

Nevalainen, J., Tempel, E., Liivamägi, L. J., et al. 2015, A\&A, 583, A142

Nicastro, F., Krongold, Y., Fields, D., et al. 2010, ApJ, 715, 854

Nicastro, F., Kaastra, J., Krongold, Y., et al. 2018, Nature, 558, 406

O'Sullivan, S. P., Machalski, J., Van Eck, C. L., et al. 2019, A\&A, 622, A16

Pfrommer, C., Springel, V., Enßlin, T. A., \& Jubelgas, M. 2006, MNRAS, 367, 113

Pinzke, A., Oh, S. P., \& Pfrommer, C. 2013, MNRAS, 435, 1061

Piro, L., den Herder, J. W., Ohashi, T., et al. 2009, Exp. Astron., 23, 67

Planck Collaboration VIII. 2013, A\&A, 550, A134

Planck Collaboration XIX. 2015, A\&A, 594, A19

Planelles, S., Mimica, P., Quilis, V., \& Cuesta-Martínez, C. 2018, MNRAS, 476, 4629

Roncarelli, M., Moscardini, L., Tozzi, P., et al. 2006a, MNRAS, 368, 74

Roncarelli, M., Ettori, S., Dolag, K., et al. 2006b, MNRAS, 373, 1339

Roncarelli, M., Pointecouteau, E., Giard, M., Montier, L., \& Pello, R. 2010, A\&A, 512, A20

Roncarelli, M., Cappelluti, N., Borgani, S., Branchini, E., \& Moscardini, L. 2012, MNRAS, 424, 1012

Roncarelli, M., Gaspari, M., Ettori, S., et al. 2018, A\&A, 618, A39

Ryu, D., Kang, H., Hallman, E., \& Jones, T. W. 2003, ApJ, 593, 599

Schmid, C., Smith, R., \& Wilms, J. 2013, SIMPUT - A File Format for Simulation Input, Tech. Report, HEASARC, Cambridge (MA)

Sheth, R., \& Tormen, G. 1999, MNRAS, 308, 119

Simionescu, A., Allen, S. W., Mantz, A., et al. 2011, Science, 331, 1576
Smith, R. K., Brickhouse, N. S., Liedahl, D. A., \& Raymond, J. C. 2001, ApJ, 556, L91

Sugawara, Y., Takizawa, M., Itahana, M., et al. 2017, PASJ, 69, 93

Takei, Y., Ursino, E., Branchini, E., et al. 2011, ApJ, 734, 91

Tanimura, H., Aghanim, N., Douspis, M., Beelen, A., \& Bonjean, V. 2019, A\&A, 625, A67

The Lynx Team 2018, ArXiv e-prints [arXiv:1809.09642]

Urban, O., Werner, N., Simionescu, A., Allen, S. W., \& Böhringer, H. 2011, MNRAS, 414, 2101

Ursino, E., Galeazzi, M., \& Roncarelli, M. 2010, ApJ, 721, 46

Ursino, E., Branchini, E., Galeazzi, M., et al. 2011, MNRAS, 414, 2970

Vacca, V., Murgia, M., Loi, F. G. F., et al. 2018, MNRAS, 479, 776

van Weeren, R. J., Williams, W. L., Hardcastle, M. J., et al. 2016, ApJS, 223, 2

van Weeren, R. J., de Gasperin, F., Akamatsu, H., et al. 2019, Space Sci. Rev., 215,16

Vazza, F., Brunetti, G., \& Gheller, C. 2009, MNRAS, 395, 1333

Vazza, F., Dolag, K., Ryu, D., et al. 2011, MNRAS, 418, 960

Vazza, F., Brüggen, M., \& Gheller, C. 2013, MNRAS, 428, 2366

Vazza, F., Ferrari, C., Bonafede, A., et al. 2015a, ArXiv e-prints [arXiv:1501.00315]

Vazza, F., Ferrari, C., Brüggen, M., et al. 2015b, A\&A, 580, A119

Vazza, F., Brüggen, M., Gheller, C., et al. 2017a, Class. Quant. Grav., 34, 234001

Vazza, F., Jones, T. W., Brüggen, M., et al. 2017b, MNRAS, 464, 210

Vazza, F., Brüggen, M., Hinz, P. M., et al. 2018a, MNRAS, 480, 3907

Vazza, F., Brunetti, G., Brüggen, M., \& Bonafede, A. 2018b, MNRAS, 474, 1672

Wang, P., Abel, T., \& Kaehler, R. 2010, New Astron., 15, 581

Werner, N., Finoguenov, A., Kaastra, J. S., et al. 2008, A\&A, 482, L29

Wittor, D., Vazza, F., \& Brüggen, M. 2017, MNRAS, 464, 4448

Zhuravleva, I. V., Churazov, E. M., Sazonov, S. Y., Sunyaev, R. A., \& Dolag, K. 2011, Astron. Lett., 37, 141 